\title{
STUDY OF ALLELIC VARIATION AT GENOME WIDE SSR LOCI IN PARENTS OF MAPPING POPULATION FOR HIGH GRAIN ZINC IN RICE (Oryza sativa L.)
}

\author{
Sonali Habde*, S. K. Singh, Korada Mounika, Amrutlal Khaire, D. K. Singh, Prasanta Kumar Majhi \\ Department of Genetics and Plant Breeding, Institute of Agricultural Sciences, Banaras Hindu University, Varanasi, Uttar Pradesh
}

Received - May 13, 2020; Revision - August 31, 2020; Accepted - September 17, 2020

Available Online - October 25, 2020

DOI: http://dx.doi.org/10.18006/2020.8(5).558.575

KEYWORDS
Rice
Quantitative trait loci (QTL)
Polymorphism survey
SSRs
Grain zinc
Mapping population

\begin{abstract}
Rice (Oryza sativa L.) is the source of energy and nutrition for more than half of the world's population hence it is a crop of global significance. Breeding of mineral dense rice varieties is the main target of biofortification strategy to address micronutrient malnutrition globally. Landraces and local genotypes are proven sources of novel alleles and are a promising donor for high grain mineral. A parental polymorphism survey is a prerequisite of any QTL mapping experiment. Present study consisting of SSR markers based parental polymorphism survey at IRRI South Asia Hub ICRISAT, Hyderabad. Parents of mapping population viz Rajendrakasturi (short grain aromatic rice variety with low grain zinc content) and URG-30 (a local genotype from Eastern Uttar Pradesh with high grain zinc content) were screened with 1013 microsatellite markers covering the entire length of all 12 chromosomes to study allelic variation at genome-wide SSR loci. The geographical diverse origin of parents is reflected in genotypic variations in terms of polymorphism. Out of 1013 whole genome wide SSR markers screened, 294 were found to be polymorphic which resulted in $29.02 \%$ polymorphism between the two parents. The highest polymorphism was observed with chromosome $4(40.96 \%)$ whereas the lowest polymorphism was observed in chromosome $9(16 \%)$. Based on the outcomes of the present study, a set of genome-wide polymorphic SSRs will be selected for genotyping of mapping population, preparation of linkage map and QTL analysis for high grain zinc content, iron content, grain quality and yield traits.
\end{abstract}

* Corresponding author

E-mail: sonali.habde@gmail.com (Sonali Habde)

Peer review under responsibility of Journal of Experimental Biology and Agricultural Sciences.

Production and Hosting by Horizon Publisher India [HPI] (http://www.horizonpublisherindia.in/).

All rights reserved.
All the articles published by Journal of Experimental Biology and Agricultural Sciences are licensed under a Creative Commons Attribution-NonCommercial 4.0 International License Based on a work at www.jebas.org.

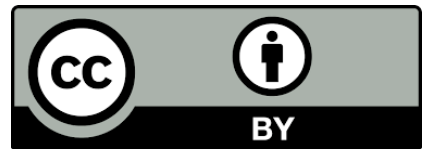




\section{Introduction}

Rice is the world's most important crop and more than half of the global population is dependent on rice (Rao et al., 2020). Rice is the chief source of carbohydrate and is used as an integral part of a balanced diet for billions of people in the world (Das et al., 2020). So it is a crop of global significance. In the Indian context, rice has deeply embedded itself in Indian culture. Although the country has attained self-sufficiency in rice production (Ghose et al., 2013) but nutritional enrichment of rice with enhanced grain quality, without compromise on yield and consumer acceptance is a new challenge ahead for the plant breeders. Even though rice is rich in starch $(80 \%)$ but it is deficient in major micronutrients such as zinc and iron. Zinc $(\mathrm{Zn})$ is one of the essential micronutrients for human health which plays an important role in the normal functioning of the immune system, reproductive growth, nervous system development and behavior (Hotz \& Brown, 2004). Iron (Fe) and Zinc $(\mathrm{Zn})$ deficiencies are among the most prevalent micronutrient deficiencies in humans and one-third of the world's population are at the risk of zinc deficiency (HarvestPlus, 2014). Dietary deficiency of iron and zinc is identified as a major cause of malnutrition (Bouis \& Saltzman, 2017). Micronutrient deficiencies have raised health risk in developing countries and also its existing spectrum has expanded to those strata of society who is dependent on the unbalanced diet of single staple food rice hence impacting global public health (Virk \& Barry, 2009).

Biofortification is the process of enriching food grains for nutritive elements in crop plants using genetic approaches (Pradhan et al., 2020a). The biofortification strategy implemented with the aids of breeding will help to address the problem of micronutrient malnutrition in a more sustainable way (Bouis \& Welch, 2010). The precision breeding approach is recommended for nutritional enhancement of high yielding varieties with major micronutrients which includes the use of potential donors, identification of QTLs for high grain mineral content, and identification of robust markers (Pradhan et al., 2020b). Dissecting genomic complexity by mapping quantitative trait loci (QTLs) for grain quality traits, for their deployment in marker-assisted selection and gene discovery is an effective forward genetic approach (Swamy et al., 2012). To design an effective breeding strategy of rice biofortification, identification of genomic regions associated with grain iron and zinc content is essential (Bollinedi et al., 2020). Improvement of any trait in rice has always benefited to a sizable proportion of the population.

In this context, plant breeders can play a major role in screening genetic variability in the trace mineral concentration, identification of genetic basis and genomic region of complex polygenic traits, perform target trait mapping and marker-assisted selection for the improvement of grain quality and nutritional traits. SSRs marker always remains the choice of plant breeders, due to their abundance, genome coverage, low analysis cost, co-dominant nature, high reproducibility, high level of polymorphism and their high utility in marker assisted selection.

Although there are various markers systems available in rice, microsatellites or simple sequence repeats (SSRs) have high polymorphism and are technically simple for detection hence are widely used (McCouch et al., 2002). SSRs have been used widely in the majority of crop species including rice for which sequence information is available. These are mainly useful for gene tagging, genetic diversity studies, genotyping of mapping population, linkage map preparation, tracing marker-trait association, singlemarker analysis and all types of QTL mapping analysis. Generally, there are two types of SSRs viz Class I and Class II. Class I SSRs also called hypervariable markers have longer repeats of $\geq 20$ nucleotides in length hence are more polymorphic than Class II SSRs (Cho et al., 2000; Temnykh et al., 2000).

Parental polymorphism survey is a prerequisite for every QTL mapping experiment. Hence the present study was conducted to screen genome wide distributed large number of SSR markers between two parental genotypes of rice viz Rajendrakasturi and URG-30. The study was aimed to identify the polymorphic markers set for allelic variation between both parents, to study their distribution across the chromosomes and identify repeat motif. Based on these, a subset of SSR will be selected and utilized for performing QTL mapping in mapping population developed from the above cross between these two parents for various traits of interest like nutritional trait involving high grain zinc content, high grain iron content; grain quality traits and yield trait. As per phenotypic evaluation of parents a higher amount of variation was observed for enough traits like yield, yield-related traits, quality and nutritional traits. So, there is huge scope for QTL mapping analysis for the various number of traits in mapping population developed from the cross of both the parents.

\section{Materials and Methods}

\subsection{Selection of Parents for Study}

The two parents viz Rajendrakasturi and URG-30 were selected for this study, among these Rajendrakasturi is short grain aromatic variety which developed from Kasturi $\times$ Sungadha parentage by Dr. Rajendra Prasad Central Agricultural University, Pusa, Bihar. This was selected for its quality traits while URG-30 is a genotype from Eastern Uttar Pradesh collected by the Institute of Agricultural Sciences, Banaras Hindu University, Varanasi as local collection material and identified and considered by the Harvest Plus project as stable high zinc donor with high grain iron content. Both selected parents have contrasting features for desirable grain quality and nutritional traits. Details of all the traits of both the parents have been mentioned in table 1 . The mapping population was developed by crossing both parents and advancing they confirm $F_{1}$ plant to raise $F_{2}$ population. 
Table 1 Characteristics features of parental lines; two parents used for present study Rajendra kasturi and URG-30 used in the experiment S.No. Trait name Rajendrakasturi URG-30

\begin{tabular}{|c|c|c|c|}
\hline 1. & Source & $\begin{array}{c}\text { Available in project material at Institute of } \\
\text { Agricultural Sciences, BHU, Varanasi, U.P, India. }\end{array}$ & $\begin{array}{l}\text { Upland rice genotype collected as Local } \\
\text { germplasm by Institute of Agricultural } \\
\text { Sciences, BHU, Varanasi, U.P, India. }\end{array}$ \\
\hline 2. & Parentage & Kasturi $\times$ Sungadha & Upland rice genotypes \\
\hline 3. & Release year and Centre & $\begin{array}{l}2004 \text { by Dr. Rajendra Prasad Central Agricultural } \\
\text { University, Pusa, Bihar }\end{array}$ & $\begin{array}{c}\text { Included in Harvest Plus Project (High } \\
\text { zinc donor) }\end{array}$ \\
\hline 4. & Yield & $40-42 \mathrm{q} / \mathrm{ha}$ & $28-30 \mathrm{q} / \mathrm{ha}$ \\
\hline 5. & Days to maturity & $125-130$ days & 108-110 days \\
\hline 6. & Plant Height & $105 \mathrm{~cm}$ & $101 \mathrm{~cm}$ \\
\hline 7. & Brown rice Zinc content & $17-19 \mathrm{ppm}$ & $30-33 \mathrm{ppm}$ \\
\hline 8. & Aroma & Present & Absent \\
\hline 9. & Kernel length & $4.0 \mathrm{~mm}$ & $5.02 \mathrm{~mm}$ \\
\hline 10. & Kernel width & $1.9 \mathrm{~mm}$ & $2.4 \mathrm{~mm}$ \\
\hline 11. & Kernel color & White & Red \\
\hline 12. & Panicle type & Compact & Spreading \\
\hline 13. & Seed shattering & Absent & Present \\
\hline 14. & Awns & Absent & Present (small) \\
\hline
\end{tabular}

\subsection{Field trial and leaf sample collection}

Present experiment was conducted during Rabi-2018 at IRRI South Asia Hub, ICRISAT, Hyderabad. Both parents viz Rajendrakasturi and URG-30 were raised by following all recommended package of practices of the region. Leaf samples of both the parents were collected from 21 days old seedlings which were transplanted in the field later for evaluation. Collected leaf samples were preserved at $-20^{\circ} \mathrm{C}$ up to further use. Both parents were evaluated for morphological and yield-related traits. Collected leaf samples were used for DNA isolation for the present study.

\subsection{DNA isolation}

Parental genomic DNA isolation was done by a quick DNA extraction method (TPS Buffer) adopted from Dr. Yohie Koide for IRRI-JAPAN Collaborative Research Project and modified from Lenie A. Quiatchon (Kim et al., 2016). Micro Tubes of $1.1 \mathrm{ml}$ were added with fresh leaf samples (around $100 \mathrm{mg}$ ), two steel beads and $600 \mu \mathrm{l}$ of TPS buffer (100mM Tris $\mathrm{HCl} \mathrm{pH} 8.0,10 \mathrm{mM}$ EDTA, $1 \mathrm{M}$ $\mathrm{KCl}$, nano pure water for volume makeup). Micro Racks sealed properly and subjected to Geno Grinder with speed $900 \mathrm{rpm}$ set for 3 min. (repeat this 3-4 times) and incubated in a water bath at $55^{\circ} \mathrm{C}$ for $30 \mathrm{~min}$. Then samples were centrifuged for $30 \mathrm{~min}$, at $3000 \mathrm{rpm}$ and the supernatant was collected in a fresh tube (96 Eppendorf deep well storage microplate). Chilled isopropanol @ $300 \mu 1$ was added to each tube and kept in the refrigerator at $-20^{\circ} \mathrm{C}$ for overnight. DNA pellet was collected by centrifuge at $3000 \mathrm{rpm}$ for $30 \mathrm{~min}$ at $4^{\circ} \mathrm{C}$ and washed with $160 \mu 170 \%$ ethanol and centrifuge at 2000 rpm for $20 \mathrm{~min}$. Pouring off ethanol, pellets were allowed to dry up completely and rehydrated with $100 \mu \mathrm{l}$ of $1 \mathrm{X}$ TE buffer (1M Tris pH-8.0, 0.5M EDTA pH- 8.0).

\subsection{Quantification of Genomic DNA and Agarose gel electrophoresis.}

The quality of DNA was evaluated by running it on $0.8 \%$ agarose gel along with a standard ladder and compared with band intensity and thickness. $0.8 \%$ Agarose $(800 \mathrm{mg}$ ) was added to $100 \mathrm{ml}$ of $1 \mathrm{X}$ TAE buffer melted till it became transparent. The solution was cooled to about $50^{\circ} \mathrm{C}$, added with $6 \mu \mathrm{l}$ of ethidium bromide $(0.5 \mu \mathrm{g}$ $\mathrm{mL}^{-1}$ ), and poured into the gel casting unit. $2 \mu 1$ of DNA samples added with gel loading dye were electrophoresed (GeNei horizontal mini gel unit) in agarose gel at 60 Volts in TAE buffer for $1 \mathrm{hr}$. After electrophoresis, the band intensity of genomic DNA was visualized on the Gel documentation unit (SYNGENE GBox, UK) (Yerva et al., 2018). These gels provided a visual measure of purity and integrity of DNA. 


\subsection{Use of Rice Microsatellite Markers for the parental polymorphism survey}

A total of 1013 microsatellite markers (IDT ${ }^{\circledR}$ ) covering the entire length of each of 12 chromosomes of rice were used for this study. In this study, it is ensured that marker distribution will be uniform with whole-genome coverage across all chromosomes. The details about McCouch Locus_ID, physical position (SSR start and SSR end), number of repeat motif, forward and reverse primer sequences, chromosome number, etc., were obtained from the Gramene markers database (https://www.gramene.org/).

\subsection{Use of RAP-DB}

For those markers whose information was not available in the Gramene database, it was obtained from RAP-DB (The Rice Annotation Project DataBase) by using its BLAST tool and submitting query sequence in FASTA format to run a search against DNA database (blastn) (https://rapdb.dna.affrc.go.jp/tools/blast).

\subsection{PCR amplification of genomic DNA using SSR markers}

DNA amplification using SSR markers was carried out with Polymerase chain reaction in Eppendorf nexus gradient master cycler. PCR reaction was done in $0.2 \mathrm{ml} 96$ well PCR plates (Watson $137-174 \mathrm{C}$ ) by adding $1 \mu \mathrm{Taq}$ buffer $\left[\mathrm{MgCl}_{2}: 100 \mathrm{mM}\right.$ Tris ( $\mathrm{pH}$ 9.0) $500 \mathrm{mM} \mathrm{KCl}, 15 \mathrm{mM} \mathrm{MgCl}_{2}$, and $0.1 \%$ gelatin], forward and reverse primer each $0.5 \mu 1$ (10 picomoles each), $3 \mu 1$ of genomic DNA, $0.5 \mu \mathrm{l}$ of dNTPs $(2.5 \mathrm{mM}), 0.1 \mu \mathrm{l}$ Taq DNA polymerase 3U/ $\mu 1$ (Bangalore GeNei Laboratories Private Limited) and $4.4 \mu 1$ of sterilized distilled water to make a final volume of $10 \mu 1$ for each reaction (Yerva et al., 2018). PCR plate kept in a thermal cycler and the reaction was set as $1^{\text {st }}$ cycle initial denaturation at $94^{\circ} \mathrm{C}$ for $5 \mathrm{~min}$, followed by 35 cycles of denaturation at $94^{\circ} \mathrm{C}$ for 30 seconds, annealing at $58^{\circ} \mathrm{C}$ (touch down $-0.1^{\circ} \mathrm{C} /$ cycle) for 30 seconds and primer extension at $72^{\circ} \mathrm{C}$ for 1 minute. A final extension was given at $72^{\circ} \mathrm{C}$ for $5 \mathrm{~min}$ at the end of the cycle and samples were held at $4^{\circ} \mathrm{C}$ till retrieval. PCR product was analyzed on 3\% agarose gel added with Ethidium bromide and standard DNA ladders of $50 \mathrm{bp}$ and $100 \mathrm{bp}$ was then visualized under the Gel Doc system. Band pattern was observed and polymorphic markers were recorded.

\subsection{Use of GGT 2.0 (software for display of Graphical Genotypes)}

Graphical representation of marker data was obtained with GGT 2.0 software (Van Berloo, 2008). GGT 2.0 deals with visualization and analyses that involve molecular marker scores (Van Berloo, 2008). Based on inputs of physical positions of markers given in row and column data matrix, visualization of the distribution of polymorphic markers across the length of chromosome according to their physical positions $(\mathrm{Mb})$ was obtained by GGT represented in figure 1 (Van Berloo, 2007; Van Berloo, 2008).

\section{Results and Discussion}

In the present investigation, only those SSRs which showed clear polymorphism in terms of scorable band difference were considered (Figure 4). Out of 1013 SSR markers screened, 294 SSRs were polymorphic indicating overall polymorphism of 29.02\% between the two parents used for the study. At the individual chromosome level, the highest polymorphism was observed with chromosome $4(40.96 \%)$ followed by chromosome $6(37.8 \%)$ whereas the lowest polymorphism was observed in chromosome $9(16 \%)$. Details of polymorphism percentage of individual chromosomes are mentioned in table 2. Further 38 polymorphic markers were present on chromosome 1 , followed by 34 polymorphic markers on chromosome 2 and 4 individually, the least number of polymorphic markers (12) were obtained for chromosome 12. A detailed list of all the polymorphic markers obtained on each chromosome is given in Table 3. Kumar et al. (2013) reported $31 \%$ polymorphism with 128 HRM primers which were screened between JGL 1798 and donor parents GPP 2 and NLR 145. Parental polymorphism survey between popular rice varieties of Andaman \& Nicobar Islands viz C14-8 and CARI Dhan 5 with donor IRBB 60 showed $36 \quad(18 \%$ polymorphism) and 48 (24\% polymorphism) polymorphic markers out of 200 SSR markers screened and the highest polymorphism was of 53\% reported for chromosome 4 (Gautam et al., 2015). Parental polymorphism survey between the two parents (N22 and Uma) revealed 20.82 percent polymorphism with 41 polymorphic markers out of 197 markers screened (Waghmare et al., 2018). Another study using parental lines PR122 and IR10M196 surveyed 647 SSR markers and found 108 polymorphic markers with $16.69 \%$ of polymorphism level between parents (Yerva et al., 2018). A polymorphism survey conducted by Challa \& Kole (2019) using drought contrasting parents reported overall polymorphism of $13.17 \%$ in which the highest polymorphism was noticed in chromosome 5 (17.02\%) and the lowest was noticed in chromosome 10 (5.36\%). Parental polymorphism in iron and zinc rich rice varieties 52 polymorphic markers were obtained out of 171 total screened rice microsatellite markers (Shivani et al., 2020). In another study by Vishalakshi et al. (2020) also assess the polymorphism and reported that, out of 500 markers screened between Varalu and Vandana NIL, 150 polymorphic markers was reported which expressed $30 \%$ polymorphism. Out of this highest polymorphism was recorded for chromosome $12(42.5 \%)$ and least for chromosome 8 (20\%).

The necessity of combining different genetic backgrounds for QTL mapping experiment for grain zinc and iron content has been also highlighted by Lee et al. (2020). A higher level of polymorphism in the present study is a result of the diverse 
Legend $\nabla^{A}$

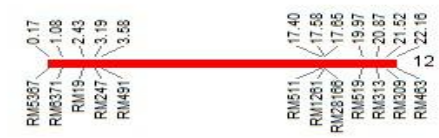

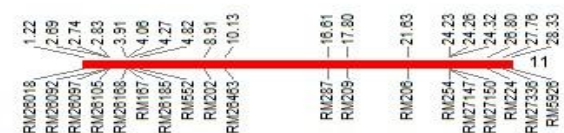

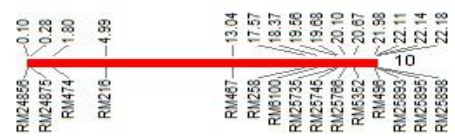

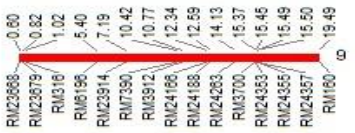

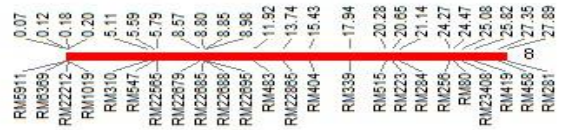

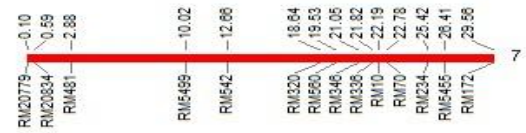

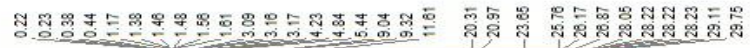

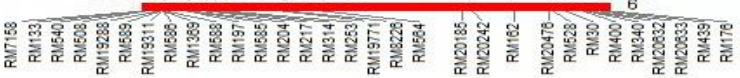

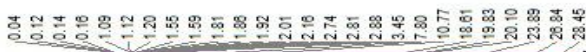

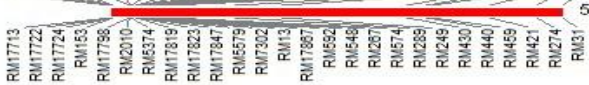

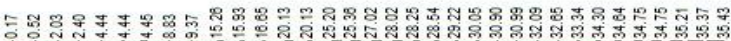

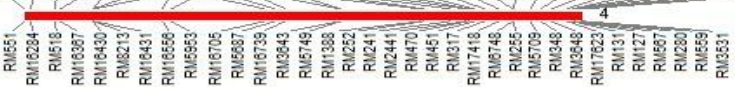

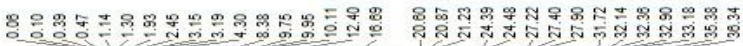

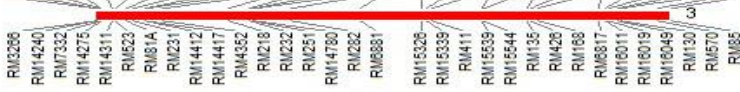

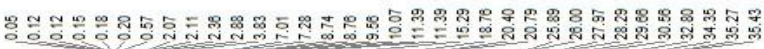

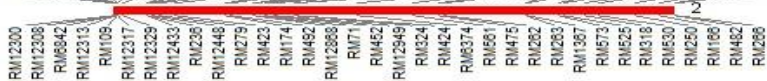

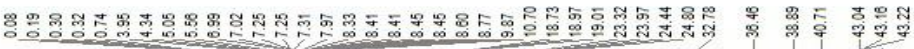

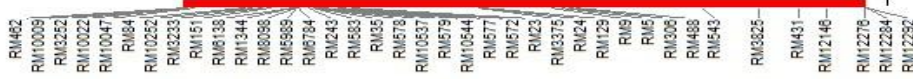


Table 2 Details of study of chromosome wise polymorphism percentage of SSRs markers between the parents Rajendrakasturi and URG-30

\begin{tabular}{|ccccc|}
\hline Chromosome no. & Total No. of SSR marker run & No. of Polymorphic markers & Polymorphism (\%) \\
\hline 1 & 138 & 38 & 35.41 \\
\hline 2 & 96 & 34 & 29.90 \\
\hline 3 & 107 & 32 & 40.96 \\
\hline 4 & 83 & 34 & 31.70 \\
\hline 5 & 82 & 26 & 37.80 \\
\hline 6 & 82 & 31 & 24.56 \\
\hline 7 & 57 & 14 & 29.62 \\
\hline 8 & 81 & 24 & 16.00 \\
\hline 9 & 96 & 15 & 27.77 \\
\hline 10 & 54 & 15 & 21.59 \\
\hline 11 & 88 & 19 & 24.48 \\
\hline 12 & 49 & 12 & 29.02 \\
\hline Total & 1013 & 294 & \\
\hline
\end{tabular}

Table 3 List of all the polymorphic markers obtained on 12 chromosomes with their details

\begin{tabular}{|c|c|c|c|c|c|c|c|}
\hline S. N & $\begin{array}{c}\text { Name of } \\
\text { SSR } \\
\text { marker }\end{array}$ & $\begin{array}{l}\text { Chr. } \\
\text { No }\end{array}$ & $\begin{array}{l}\text { Physical } \\
\text { position } \\
(\mathrm{Mb})\end{array}$ & Motif & $\begin{array}{l}\text { Number } \\
\text { of } \\
\text { Repeats }\end{array}$ & Forward Primer Sequence & Reverse Primer Sequence \\
\hline 1 & RM462 & 1 & 0.08 & AG & 12 & CCGCGAATCCATTCAGACTGC & TCTAGGAGGAGATGGCGGAGTAGC \\
\hline 2 & RM10009 & 1 & 0.19 & AG & 31 & GATGCTCCGGAATAACTAGATTGG & GGAATTACAGCTGTCTTGGAAGG \\
\hline 3 & RM3252 & 1 & 0.3 & AG & 13 & ATGCAAGCATCTGCTTATGG & GTTGGTAACTTTGTTCCCATGC \\
\hline 4 & RM10022 & 1 & 0.32 & AAAG & 5 & CCTCCATAGAGTAAGGTTTGCATGG & СCTCCTCCTCTGTCTTTCTCTGC \\
\hline 5 & RM10047 & 1 & 0.74 & AC & 13 & CAAGCAGTGATCATACAGCCTTCC & GCCATGGCTGAGAACAGAGAGC \\
\hline 6 & RM84 & 1 & 3.95 & TCT & 10 & TAAGGGTCCATCCACAAGATG & TTGCAAATGCAGCTAGAGTAC \\
\hline 7 & RM10252 & 1 & 4.34 & ACAT & 5 & GGTGAGATACTTATTCGTGAGTGC & AACCGCTTACCATGATAGTACC \\
\hline 8 & RM3233 & 1 & 5.05 & AG & 13 & GAAATTCGAAATGGAGGGAGAGC & GGTGAGTAAACAGTGGTGGTGAGC \\
\hline 9 & RM151 & 1 & 5.56 & AT & 44 & TGCTGATCAGTTACACGAATCAGAGC & GCGTACGTGCACAAATTAAACAGACC \\
\hline 10 & RM6138 & 1 & 6.99 & CCG & 9 & GACTCGTTCAAGACGTGGTCAGG & ACTCTCGGCATCGACGAGTACG \\
\hline 11 & RM1344 & 1 & 7.02 & AG & 23 & AATGTTCCTGCAATCCGAGTAGG & CACAGAATGTGAGCATTCACTCC \\
\hline 12 & RM8098 & 1 & 7.25 & AT & 26 & TTCGTATCCTGATACCGTTACGC & GGCCTTGATGAACTTGATTTCG \\
\hline 13 & RM5989 & 1 & 7.25 & $\mathrm{ACC}$ & 9 & CTGCGAAGTAGGTGCCCTTTGC & TCCTTCAAACTCATCACCCATTCG \\
\hline 14 & RM6784 & 1 & 7.31 & ACG & 8 & TACCACCTACCACCACGATGACG & GTAGACGACTAGCTCGCCCATCC \\
\hline 15 & RM243 & 1 & 7.97 & CT & 18 & GATCTGCAGACTGCAGTTGC & AGCTGCAACGATGTTGTCC \\
\hline 16 & RM583 & 1 & 8.33 & AAG & 20 & GTTGCGGTTTGTTCGTTCTTGC & TAGATCCCAGCAGACGGATCAGC \\
\hline 17 & RM577 & 1 & 8.37 & TA,CA & 9,8 & GCTTTCCCTCTAACCCCTCT & GGATGTACCGCTGACATGAA \\
\hline 18 & RM35 & 1 & 8.41 & GA & 19 & TGGTTAATCGATCGGTCGCC & CGACGGCAGATATACACGG \\
\hline 19 & RM578 & 1 & 8.41 & GA & 19 & GGCGTCGTGTTTTCTCTCTC & CAAAAAGGAGGAGCAGATCG \\
\hline 20 & RM10537 & 1 & 8.45 & AAG & 9 & ATTGCGGTAACCTAAGTAGCATCC & CGACACTAGCTACCAACATGTCC \\
\hline
\end{tabular}

Journal of Experimental Biology and Agricultural Sciences http://www.jebas.org 


\begin{tabular}{|c|c|c|c|c|c|c|c|}
\hline S. N & $\begin{array}{l}\text { Name of } \\
\text { SSR } \\
\text { marker }\end{array}$ & $\begin{array}{l}\text { Chr. } \\
\text { No }\end{array}$ & $\begin{array}{c}\text { Physical } \\
\text { position } \\
(\mathrm{Mb})\end{array}$ & Motif & $\begin{array}{c}\text { Number } \\
\text { of } \\
\text { Repeats }\end{array}$ & Forward Primer Sequence & Reverse Primer Sequence \\
\hline 21 & RM579 & 1 & 8.45 & GA & 25 & TCCGAGTGGTTATGCAAATG & AATTGTGTCCAATGGGCTGT \\
\hline 22 & RM10544 & 1 & 8.6 & AGC & 9 & GGGCGAAACGGAAAGAGTAGACG & TCCTACTACTCGTGAGGCGAGAGG \\
\hline 23 & RM572 & 1 & 9.87 & AG & 14 & CGCGGTTAATGTCATCTGATTGG & CCATACTTCGAGATCCAAGACTGACC \\
\hline 24 & RM23 & 1 & 10.7 & GA & 15 & CATTGGAGTGGAGGCTGG & GTCAGGCTTCTGCCATTCTC \\
\hline 25 & RM3375 & 1 & 18.73 & AG & 16 & TGCAAGGAAACTAGGAGGGAAGC & CTTGACCTCCTCCTCCACAACC \\
\hline 26 & RM24 & 1 & 18.97 & GA & 29 & GAAGTGTGATCACTGTAACC & TACAGTGGACGGCGAAGTCG \\
\hline 27 & RM129 & 1 & 19.01 & CGG & 8 & TCTCTCCGGAGCCAAGGCGAGG & CGAGCCACGACGCGATGTACCC \\
\hline 28 & RM9 & 1 & 23.32 & AG & 14 & GGCCCTCATCACCTTCGTAGC & СGTCCTCCCTCTCCCTATCTCC \\
\hline 29 & RM5 & 1 & 23.97 & GA & 14 & TGCAACTTCTAGCTGCTCGA & GCATCCGATCTTGATGGG \\
\hline 30 & RM306 & 1 & 24.44 & $\begin{array}{l}\text { GT,AT, } \\
\text { CT,GT }\end{array}$ & $18,8,1,6$ & CAAGGTCAAGAATGCAATGG & GCCACTTTAATCATTGCATC \\
\hline 31 & RM488 & 1 & 24.8 & AG & 17 & AACAACCAGCGTATGCGTTCTCG & CCCACGGCTTTGTAGGAAGAAGC \\
\hline 32 & RM543 & 1 & 32.78 & GCG & 10 & CTGCTGCAGACTCTACTGCG & AAATATTACCCATCCCCCCC \\
\hline 33 & RM3825 & 1 & 36.46 & GA & 21 & AAAGCCCCCAAAAGCAGTAC & GTGAAACTCTGGGGTGTTCG \\
\hline 34 & RM431 & 1 & 38.89 & AG & 16 & GCTTGCTTGTATCTGCATTGGTAGG & GGGATGATCCACTCTCTGTTTGG \\
\hline 35 & RM12146 & 1 & 40.71 & AG & 11 & AGTATGCCCTGCCCACTACACTAGG & CAGCGAATGGCAAGAGCAACC \\
\hline 36 & RM12276 & 1 & 43.04 & TC & 17 & GTCGACGGCTTCCTCAAGATTGG & TGAGACCTCTGTGAAGGCACTCG \\
\hline 37 & RM12284 & 1 & 43.16 & AAG & 9 & CAGCACGAACAGACAGGATGG & AGGAAGAAGAAGCAGCGATTCC \\
\hline 38 & RM12292 & 1 & 43.22 & AT & 38 & ATGAGACGATGAAAGCCTCAAGC & GTGGGACAAGCAAATTGAAACG \\
\hline 39 & RM12300 & 2 & 0.05 & AG & 14 & AGTCAGGCAGACAACACCATCG & TGCTGTTCATCTGTGTCAGTCAGC \\
\hline 40 & RM12308 & 2 & 0.12 & AG & 10 & GTTTGCGGATGCATTCAGTACC & GGATCGCCCTGCATATTTATACC \\
\hline 41 & RM6842 & 2 & 0.12 & AAG & 21 & CCGTGCATCTCGCTACCTAACC & TGCACACACAACTTAGAGGAAGAAGG \\
\hline 42 & RM12313 & 2 & 0.15 & AATG & 5 & CCACAACGCATGCTACTCTCAGC & ATGCAAGATGGTGGATGGATGG \\
\hline 43 & RM109 & 2 & 0.18 & AG & 16 & GCCGCCGGAGAGGGAGAGAGAG & CCCCGACGGGATCTCCATCGTC \\
\hline 44 & RM12317 & 2 & 0.2 & AAAG & 10 & ATAATTGTGGTCCCGAGTCATCC & TGCTTCTTCAACTCAACTCTCAGC \\
\hline 45 & RM12329 & 2 & 0.57 & AG & 15 & AGGAAGAGGCGAAGGTAGATCG & CCAATCATGCTGTGTTTCAAGG \\
\hline 46 & RM12433 & 2 & 2.07 & AG & 18 & CTCTACACGCTTTGCCTGTTTGG & TGATCAGGTTCGAGATTGGGTATGG \\
\hline 47 & RM236 & 2 & 2.11 & CT & 18 & GCGCTGGTGGAAAATGAG & GGCATCCCTCTTTGATTCCTC \\
\hline 48 & RM12448 & 2 & 2.36 & AT & 28 & CAATGGGCGGGTTTGACAGG & ACCACTTTGCTGTTCACGTCTCG \\
\hline 49 & RM279 & 2 & 2.88 & GA & 16 & GCGGGAGAGGGATCTCCT & GGCTAGGAGTTAACCTCGCG \\
\hline 50 & RM423 & 2 & 3.83 & TTC & 9 & AGCACCCATGCCTTATGTTG & CCTTTTTCAGTAGCCCTCCC \\
\hline 51 & RM174 & 2 & 7.01 & AAG & 7 & ATAAGCGACGCCAAGACAAGTCG & GGAAGCAAGAAGGAAAGAGAGATGG \\
\hline 52 & RM492 & 2 & 7.28 & AG & 21 & CATTGACGTGGCACTTTGTTCC & AGAGAGCACGCAATGGAGTATGC \\
\hline 53 & RM12868 & 2 & 8.74 & ATC & 7 & ACTCATCAACATCGCGAAACAGC & CTGACTCCGACAACAGCTATGAACC \\
\hline 54 & RM71 & 2 & 8.76 & $\begin{array}{c}\text { ATT,T, } \\
\text { ATT }\end{array}$ & $10,1,4$ & CTAGAGGCGAAAACGAGATG & GGGTGGGCGAGGTAATAATG \\
\hline 55 & RM452 & 2 & 9.56 & ACG & 9 & GTGGACTTGGCGAGATGCTACG & GTTAAGGGCAGCCACCAGATCG \\
\hline 56 & RM12949 & 2 & 10.07 & AG & 10 & CTACGCCACCAAACCCACTCC & ATTTGATGCTGTGAGGCTGTGG \\
\hline 57 & RM324 & 2 & 11.39 & ATC & 9 & GATTCCACGTCAGGATCTTCTGG & GCTCACCAGTTGAGATTGAAAGG \\
\hline 58 & RM424 & 2 & 11.39 & CAT & 9 & TTTGTGGCTCACCAGTTGAG & TGGCGCATTCATGTCATC \\
\hline
\end{tabular}




\begin{tabular}{|c|c|c|c|c|c|c|c|}
\hline S. N & $\begin{array}{c}\text { Name of } \\
\text { SSR } \\
\text { marker }\end{array}$ & $\begin{array}{l}\text { Chr. } \\
\text { No }\end{array}$ & $\begin{array}{c}\text { Physical } \\
\text { position } \\
(\mathrm{Mb})\end{array}$ & Motif & $\begin{array}{c}\text { Number } \\
\text { of } \\
\text { Repeats }\end{array}$ & Forward Primer Sequence & Reverse Primer Sequence \\
\hline 59 & RM6374 & 2 & 15.29 & AAG & 16 & TCACCAGACTCAACAAAGGATCG & ТTСАССТTТСТTСТСССТСАТTСC \\
\hline 60 & RM561 & 2 & 18.76 & GA & 11 & GAGCTGTTTTGGACTACGGC & GAGTAGCTTTCTCCCACCCC \\
\hline 61 & RM475 & 2 & 20.4 & TATC & 8 & CCTCACGATTTTCCTCCAAC & ACGGTGGGATTAGACTGTGC \\
\hline 62 & RM262 & 2 & 20.79 & CT & 16 & САTTCCGTCTCGGCTCAACT & CAGAGCAAGGTGGCTTGC \\
\hline 63 & RM263 & 2 & 25.89 & CT & 34 & CCCAGGCTAGCTCATGAACC & GCTACGTTTGAGCTACCACG \\
\hline 64 & RM1367 & 2 & 26 & AG & 27 & GTGTGTACGTAGGATCGGAG & TGCTACTCCTAGCTGCTACC \\
\hline 65 & RM573 & 2 & 27.97 & AG & 11 & TCATGTTGACGCACACATACACG & СTCTTCTTCCCTGGACCACACC \\
\hline 66 & RM525 & 2 & 28.29 & AAG & 12 & GTTGGTATTCCCTGATGAGTTTGC & GAGACAGAATCCTTACGCTGTGC \\
\hline 67 & RM318 & 2 & 29.66 & AC & 11 & GGCTACGCTCCAGTTAGTGAAGG & TGGTAGGAAGAAGGGAGAAGTTTAGG \\
\hline 68 & RM530 & 2 & 30.56 & AG & 23 & TTCTTTATTCCCTCGCACTGACC & CAATGATGCCACAAACCGTAACC \\
\hline 69 & RM250 & 2 & 32.8 & AG & 27 & GTTCAAACCAAGCTGATCACAAGC & GGCGTCAGAGTCAGAGATGAAGG \\
\hline 70 & RM166 & 2 & 34.35 & $\mathrm{~T}$ & 12 & GGTCCTGGGTCAATAATTGGGTTACC & TTGCTGCATGATCCTAAACCGG \\
\hline 71 & RM482 & 2 & 35.27 & AT & 9 & TCTGAAAGCCTGACTCATCG & GTCAATTGCAGTGCCCTTTC \\
\hline 72 & RM266 & 2 & 35.43 & GA & 19 & TAGTTTAACCAAGACTCTC & GGTTGAACCCAAATCTGCA \\
\hline 73 & RM3266 & 3 & 0.06 & AG & 13 & CTTGGAGAAGAAGAGGCTGGAAGG & GGATGATATCCACCGGGATGC \\
\hline 74 & RM14240 & 3 & 0.1 & AGAT & 11 & GCTCAAAGAATGACACCGATGC & CATGGTGAGTTTGGAGTGATTGG \\
\hline 75 & RM7332 & 3 & 0.39 & ACAT & 12 & ACACTGTACACCACACTTCAGC & CACACCAAAGGGAAATTAGG \\
\hline 76 & RM14275 & 3 & 0.47 & AT & 32 & GAAACAGTTGAGAGCGGTTACTGG & GAACCACTAGATGAATGAAGGCTAGG \\
\hline 77 & RM14311 & 3 & 1.14 & TA & 37 & AGCTTGACTTCACACCCACTCG & GGACGACCTACCGATCAAGACG \\
\hline 78 & RM523 & 3 & 1.3 & AG & 14 & TGAATTCTTGCACATGGTCAGC & TGGGAGGTTTGCTAGGGTAATCC \\
\hline 79 & RM81A & 3 & 1.93 & TCT & 10 & GAGTGCTTGTGCAAGATCCA & CTTCTTCACTCATGCAGTTC \\
\hline 80 & RM231 & 3 & 2.45 & CT & 16 & CCAGATTATTTCCTGAGGTC & CACTTGCATAGTTCTGCATTG \\
\hline 81 & RM14412 & 3 & 3.15 & AT & 17 & CCCAATCGGAATTCAATCACTCC & CCATGAGGGCTCGACTGTGG \\
\hline 82 & RM14417 & 3 & 3.19 & AG & 25 & GCCGATAATTCAGAGAAGAGATGAGC & GTCTCTCGGAGTACAGCCACAGC \\
\hline 83 & RM4352 & 3 & 4.3 & AT & 17 & AATGCAAATGGCAACCAACAGC & GATTGACGCCACAACATGAACG \\
\hline 84 & RM218 & 3 & 8.38 & AG & 24 & TCAAACCAAGGTCCTTCAACTGC & TTTCTTCCACCGTCCATGTATCC \\
\hline 85 & RM232 & 3 & 9.75 & CT & 24 & CCGGTATCCTTCGATATTGC & CCGACTTTTCCTCCTGACG \\
\hline 86 & RM251 & 3 & 9.95 & CT & 29 & GAATGGCAATGGCGCTAG & ATGCGGTTCAAGATTCGATC \\
\hline 87 & RM14780 & 3 & 10.11 & AG & 25 & GCAACCTGTTCTCGCTGAAGTAGC & GCCATTGGAGGATCCAAGATAAGC \\
\hline 88 & RM282 & 3 & 12.4 & GA & 15 & CTGTGTCGAAAGGCTGCAC & CAGTCCTGTGTTGCAGCAAG \\
\hline 89 & RM6881 & 3 & 16.69 & ACC & 11 & CGACTGATTCGATTCCACAATGATCC & GCAGCTGGAAGCAGAGGAAGACG \\
\hline 90 & RM15326 & 3 & 20.6 & AT & 29 & TGAATCTACCGCTCTACTTGTGG & AAACAGTGCATCCTTCTTGTGG \\
\hline 91 & RM15339 & 3 & 20.87 & AT & 15 & NA & NA \\
\hline 92 & RM411 & 3 & 21.23 & AAC & 8 & GTAGGAAATTCTTCGCCAGATGC & CCGAGACTTGGAACAATCTTAGGC \\
\hline 93 & RM15539 & 3 & 24.39 & AAT & 24 & CATGATGCATCGAGAATCTACCC & AAGCGTTGTGTCTACCAACATGG \\
\hline 94 & RM15544 & 3 & 24.48 & AGAT & 5 & GCTTGAGAGTGTTTGTAGGAGTGC & TTGGCAATGTACTCTGCTGTCC \\
\hline 95 & RM135 & 3 & 27.22 & CCG & 11 & TCCATGCTCTTCAGCTTCTGG & GCTTCTACTGGAGGAGAGCAGAGG \\
\hline 96 & RM426 & 3 & 27.4 & AT & 11 & CATCGCCGAAATCCATCTTCC & AAGGCCCATTTCATTGTAGAGTGC \\
\hline 97 & RM168 & 3 & 27.9 & $A C$ & 10 & TGTCGTCGAGGATTTGGAGATCG & GAATCAATCCACGGCACAGTCC \\
\hline
\end{tabular}

Journal of Experimental Biology and Agricultural Sciences http://www.jebas.org 


\begin{tabular}{|c|c|c|c|c|c|c|c|}
\hline S. N & $\begin{array}{c}\text { Name of } \\
\text { SSR } \\
\text { marker }\end{array}$ & $\begin{array}{l}\text { Chr. } \\
\text { No }\end{array}$ & $\begin{array}{c}\text { Physical } \\
\text { position } \\
(\mathrm{Mb})\end{array}$ & Motif & $\begin{array}{l}\text { Number } \\
\text { of } \\
\text { Repeats }\end{array}$ & Forward Primer Sequence & Reverse Primer Sequence \\
\hline 98 & RM6817 & 3 & 31.72 & AAG & 9 & AAGAAGAAGCGAGAGCGAGAGG & TCCTCCTCTTGACGTGTTGAGC \\
\hline 99 & RM16011 & 3 & 32.14 & AG & 23 & CACCTTCGATTTCCTTCTCATCG & CGAGTTGTACTCTGGTTGCTTCC \\
\hline 100 & RM16019 & 3 & 32.36 & AT & 44 & GGTTTGCTCTTTACACTCATGC & GTTAGGATTGGACAGCAGAAGG \\
\hline 101 & RM16049 & 3 & 32.9 & $A C$ & 12 & TGGAATTATGCGCAGTACATCAGC & GGAGCCCATACTCCATGTAGAACC \\
\hline 102 & RM130 & 3 & 33.18 & AG & 10 & CGAGAAGAAGGAGCCCATCTCG & GCGAAGCGAAGAGGAGCAAGG \\
\hline 103 & RM570 & 3 & 35.38 & AG & 15 & AGAAATGGTGAAAGATGGTGCTACCG & CTGAATGTTCTTCAACTCCCAGTGC \\
\hline 104 & RM85 & 3 & 36.34 & $\begin{array}{c}\text { TGG,T } \\
\text { CT }\end{array}$ & 5,12 & CCAAAGATGAAACCTGGATTG & GCACAAGGTGAGCAGTCC \\
\hline 105 & RM551 & 4 & 0.17 & AG & 18 & СTTACTCCATTGGGCTGGAACC & TGTAGGGTGGTAAGAGATCCACTCC \\
\hline 106 & RM16284 & 4 & 0.52 & AT & 28 & ACACTGCTCGGGAGTTACTGACC & CAATAAGCAGGGCTACAACATGC \\
\hline 107 & RM518 & 4 & 2.03 & AG & 15 & AAGACACAAGCAAACAGCTCAACC & AAGCTTGCTTGGTTCAAGAGAGG \\
\hline 108 & RM16367 & 4 & 2.4 & AT & 21 & GTTCCCAACCGGAACAGTACG & CCCTTAGGCTGTGTTTGACATCC \\
\hline 109 & RM16430 & 4 & 4.44 & AGG & 8 & ССАСТССТСССАТСТССТАСТСС & GCCACCGCACTTGTCTATGC \\
\hline 110 & RM8213 & 4 & 4.44 & AG & 19 & TGTTGGGTGGGTAAAGTAGATGC & CCCAGTGATACAAAGATGAGTTGG \\
\hline 111 & RM16431 & 4 & 4.45 & AGG & 8 & ССАСТCСТCССАТСТССТАСТСC & GCCACCGCACTTGTCTATGC \\
\hline 112 & RM16556 & 4 & 8.83 & AT & 13 & TTGGACCAGGAGATCAATGAAGG & GTGCGCACACTCTTCTATGTGC \\
\hline 113 & RM5953 & 4 & 9.37 & CAC & 6 & AAACTTTCTGTGATGGTATC & ATCCTTGTCTAGAATTGACA \\
\hline 114 & RM16705 & 4 & 15.26 & AT & 22 & GGTTAAGCACTAATGGTGGTAGG & TAACTAGTCATCTCTGCTGCAACC \\
\hline 115 & RM5687 & 4 & 15.93 & AAT & 17 & AGAGAAGAAGGGAAGGAGGAAGG & AGTGACTTGTGGGTGACTTGTGG \\
\hline 116 & RM16739 & 4 & 16.65 & AG & 21 & TGCATAGTCCACCTAAGAGTTCAAGG & TTTCTGCAGGCTGATTAGTGTAGG \\
\hline 117 & RM3643 & 4 & 20.13 & AG & 14 & GCTAAGCTAATCTGACCGGATCTACG & GATGGGCCGATTAACAAATTCC \\
\hline 118 & RM5749 & 4 & 20.13 & ACT & 7 & GCTCGTTTCTCTCGATCACTCG & GCAAGGTTGGATCAGTCATTTCG \\
\hline 119 & RM1388 & 4 & 25.2 & AG & 46 & GATCGTTGCTGCTTTCAATGAGG & AGTGTTTCACCTTGGACCCATGC \\
\hline 120 & RM252 & 4 & 25.36 & CT & 19 & TTCGCTGACGTGATAGGTTG & ATGACTTGATCCCGAGAACG \\
\hline 121 & RM241 & 4 & 27.02 & СT & 31 & GAGCCAAATAAGATCGCTGA & TGCAAGCAGCAGATTTAGTG \\
\hline 122 & RM2441 & 4 & 28.02 & AT & 27 & CGCTGCCATCATCTACAACAGG & CAACAAATGCTACCTCACCATGC \\
\hline 123 & RM470 & 4 & 28.25 & AAG & 14 & CCCTCCCGTAGACCTTGTACCC & CCACAGCTAACCAATCCTTCTCC \\
\hline 124 & RM451 & 4 & 28.54 & GAT & 8 & GATCCCCTCCGTCAAACAC & СССТTСТССТTТССТСААСС \\
\hline 125 & RM317 & 4 & 29.22 & GC,GT & 4,18 & CATACTTACCAGTTCACCGCC & CTGGAGAGTGTCAGCTAGTTGA \\
\hline 126 & RM17418 & 4 & 30.05 & ACG & 10 & GCATCCCAGCATCAGAGATAACC & САTTCTTGCGTGAGAACCAAACC \\
\hline 127 & RM6748 & 4 & 30.9 & AAT & 26 & AGAGAAGCAGCTGGTGATTAGCC & CAACGATGTACCAGTTGAATACCC \\
\hline 128 & RM255 & 4 & 30.99 & AG & 19 & GAGGAGGAGGAGGAGAGATCAGG & AACGAAACCGCTCAGTTCAACC \\
\hline 129 & RM5709 & 4 & 32.09 & AAT & 24 & TTTGGGATAAATGGGAGAGG & TCAGCTGGTTATTATGGAGAGG \\
\hline 130 & RM348 & 4 & 32.65 & CAG & 7 & CCGCTACTAATAGCAGAGAG & GGAGCTTTGTTCTTGCGAAC \\
\hline 131 & RM3648 & 4 & 33.34 & AG & 14 & CGAGAAGCCGAAGAGGAAGAGAGC & CCAACAGATCCATCTCAACCAACTCC \\
\hline 132 & RM17623 & 4 & 34.3 & AG & 12 & GTTCTTGGGTTGGTGAGCTTCC & TAGGGCGCATGTGTATTTCTTCC \\
\hline 133 & RM131 & 4 & 34.64 & AG & 15 & GGAGCAGCTTCTCGAGCATGG & CCAAATCTCGCCTCGTTTAGCC \\
\hline 134 & RM127 & 4 & 34.75 & AGG & 8 & GTGGGATAGCTGCGTCGCGTCG & AGGCCAGGGTGTTGGCATGCTG \\
\hline 135 & RM567 & 4 & 34.75 & AG & 23 & GCATACCGTAATGTTGGTGAAGC & AATAGCAACTGGGAGGAGGTAAGG \\
\hline 136 & RM280 & 4 & 35.21 & AG & 11 & GTGCTCTCCATGTCGGATTATGC & CAAGGCAACAAGATTGGTTAGTGG \\
\hline
\end{tabular}

Journal of Experimental Biology and Agricultural Sciences http://www.jebas.org 


\begin{tabular}{|c|c|c|c|c|c|c|c|}
\hline S. N & $\begin{array}{c}\text { Name of } \\
\text { SSR } \\
\text { marker }\end{array}$ & $\begin{array}{c}\text { Chr. } \\
\text { No }\end{array}$ & $\begin{array}{c}\text { Physical } \\
\text { position } \\
(\mathrm{Mb})\end{array}$ & Motif & $\begin{array}{c}\text { Number } \\
\text { of } \\
\text { Repeats }\end{array}$ & Forward Primer Sequence & Reverse Primer Sequence \\
\hline 137 & RM559 & 4 & 35.37 & AAAC & 6 & AGAGCGATGGGTGTCAGTTTGC & CGTACGTACACTTGGCCCTATGC \\
\hline 138 & RM3531 & 4 & 35.43 & AG & 12 & TATCGCCACTCGTCCATTCTCC & CAGCTACCAACACCTCCATACCC \\
\hline 139 & RM17713 & 5 & 0.04 & AG & 20 & GGGCACCATTAACTTAACAACG & GCAATGGTACAAATAGCCAAGC \\
\hline 140 & RM17722 & 5 & 0.12 & AGGC & 5 & TGCAGCTGAAGAAGAAATGAGC & ATGATGGATCTTCCAACAGTGC \\
\hline 141 & RM17724 & 5 & 0.14 & AT & 30 & TGCTCTCTTGAGTACCACTGTCC & TTTGAGACGACCACGTAGAGC \\
\hline 142 & RM153 & 5 & 0.16 & AAG & 9 & CCTCGAGCATCATCATCAGTAGG & TCCTCTTCTTGCTTGCTTCTTCC \\
\hline 143 & RM17798 & 5 & 1.09 & AT & 29 & TAATAGGAAGAGTGCGTCAGAGC & TCGACCAGTGATAACCAGTAACC \\
\hline 144 & RM2010 & 5 & 1.12 & AT & 19 & GCAATACACGCCAATCTTCTAGG & TTGGGATGGAGGTAGTATTGTGC \\
\hline 145 & RM5374 & 5 & 1.2 & AG & 13 & TTAGGGTTAGGTCTGTTGTGTACTGG & CGCTGGATCACAACACTGTAGC \\
\hline 146 & RM17819 & 5 & 1.55 & AAG & 18 & TTGCCTCATGTTTGCTTCATCC & AGCTGACAACGACGACACTGC \\
\hline 147 & RM17823 & 5 & 1.59 & AT & 31 & ACATGGTCTCGCTAGATCTTTCC & СТTССТCСATGATAAGCAAAGC \\
\hline 148 & RM17847 & 5 & 1.81 & AG & 19 & ТТСТТСТСССТСТСТТАСТСТСС & СTCTAGTCTCTTCTGCAAGTTCC \\
\hline 149 & RM5579 & 5 & 1.86 & $\mathrm{AC}$ & 22 & CAACAGAACCTGCCAACTCTTGC & TGCCTCATGGTAATAAGCCAAGC \\
\hline 150 & RM7302 & 5 & 1.92 & AATT & 6 & GGGAGAGAGGAAGGAGGAGCTTCG & CGGGACCGGAACCTTTCAACG \\
\hline 151 & RM13 & 5 & 2.01 & GA & 16 & TCCAACATGGCAAGAGAGAG & GGTGGCATTCGATTCCAG \\
\hline 152 & RM17867 & 5 & 2.16 & AT & 33 & CATCGATAGAGCCAGATAGATGC & TTTCTACAGGAAGTCAGGAACG \\
\hline 153 & RM592 & 5 & 2.74 & AAT & 26 & ACCTCACCCGAATTACTGTGATATGC & GTTGAATTGCACGCGACTCTGG \\
\hline 154 & RM548 & 5 & 2.81 & CT & 12 & TCGGTGAGAAACTGAGAGTACG & AAGGAGGCCATCTCAATGTG \\
\hline 155 & RM267 & 5 & 2.88 & GA & 21 & TGCAGACATAGAGAAGGAAGTG & AGCAACAGCACAACTTGATG \\
\hline 156 & RM574 & 5 & 3.45 & AG & 11 & AAACTAGCCACGGTTTGGTAGGG & AGGGTGGCAGGGATGTAATTTCC \\
\hline 157 & RM289 & 5 & 7.8 & G, GA & 11,16 & TTCCATGGCACACAAGCC & CTGTGCACGAACTTCCAAAG \\
\hline 158 & RM249 & 5 & 10.77 & $\begin{array}{c}A G, A, A \\
G\end{array}$ & $5,2,14$ & GGCGTAAAGGTTTTGCATGT & ATGATGCCATGAAGGTCAGC \\
\hline 159 & RM430 & 5 & 18.61 & AG & 25 & GTCCCTGATCAGAAACGAGATGG & TAGGGTTGGAAGAATGCAAGACC \\
\hline 160 & RM440 & 5 & 19.83 & AAG & 22 & GGTAGGCACCAAAGAGTTTGACG & GGCATCACCTTATCCAATCACC \\
\hline 161 & RM459 & 5 & 20.1 & ATCC & 6 & CCTCCAGTATCGATCACCAAAGC & CGAAAGAAAGTCATAGCACGATGG \\
\hline 162 & RM421 & 5 & 23.89 & AGAT & 6 & GCATAGCTATCCAGAATCCATTGACC & AGACGTAGCTCAGGTGAAACATCC \\
\hline 163 & RM274 & 5 & 26.84 & $\begin{array}{c}\mathrm{GA}, \mathrm{CG} \\
\mathrm{G}\end{array}$ & $15-7,5$ & CCTCGCTTATGAGAGCTTCG & СТTСТССАТСАСТСССАTGG \\
\hline 164 & RM31 & 5 & 28.45 & AG & 20 & CGCTCCTCСАСТСTTCTCCTACC & CGTGCAGAAAGTCCATTACTCTCC \\
\hline 165 & RM7158 & 6 & 0.22 & ACAT & 12 & CATGGACTTGTTAGCAGCGTACC & ATTTCATCGATGGCCTCTACACC \\
\hline 166 & RM133 & 6 & 0.23 & CT & 8 & TTGGATTGTTTTGCTGGCTCGC & GGAACACGGGGTCGGAAGCGAC \\
\hline 167 & RM540 & 6 & 0.38 & AG & 16 & GCCTTCTGGCTCATTTATGC & CTAGGCCTGCCAGATTGAAC \\
\hline 168 & RM508 & 6 & 0.44 & AG & 17 & AGAAGCCGGTTCATAGTTCATGC & ACCCGTGAACCACAAAGAACG \\
\hline 169 & RM19288 & 6 & 1.17 & CCG & 8 & CGGAGCTGTTGCCGTTCTGC & CGATGTGCCATGTCAGGATGACC \\
\hline 170 & RM589 & 6 & 1.38 & $A C$ & 24 & GTGGCTTAACCACATGAGAAACTACC & TCACATCATTAGGTGGCAATCG \\
\hline 171 & RM19311 & 6 & 1.46 & $A C$ & 10 & TGCGGTGCTGTTCACCTACTATCG & GCACTGAAGCTGGTGCAATCG \\
\hline 172 & RM586 & 6 & 1.48 & AG & 23 & TGCCATCTCATAAACCCACTAACC & CTGAGATACGCCAACGAGATACC \\
\hline 173 & RM1369 & 6 & 1.56 & AG & 27 & CATCGATTAGCTTACATGGCAACG & ACTAGTGCGACCGTCTTCAATGG \\
\hline 174 & RM588 & 6 & 1.61 & AGC & 9 & TCTTGCTGTGCTGTTAGTGTACG & GCAGGACATAAATACTAGGCATGG \\
\hline
\end{tabular}

Journal of Experimental Biology and Agricultural Sciences http://www.jebas.org 


\begin{tabular}{|c|c|c|c|c|c|c|c|}
\hline 568 & & & & & & & Habde et al. \\
\hline S. N & $\begin{array}{l}\text { Name of } \\
\text { SSR } \\
\text { marker }\end{array}$ & $\begin{array}{l}\text { Chr. } \\
\text { No }\end{array}$ & $\begin{array}{c}\text { Physical } \\
\text { position } \\
(\mathrm{Mb})\end{array}$ & Motif & $\begin{array}{l}\begin{array}{c}\text { Number } \\
\text { of } \\
\text { Repeats }\end{array} \\
\end{array}$ & Forward Primer Sequence & Reverse Primer Sequence \\
\hline 175 & RM197 & 6 & 3.09 & ACC & 8 & ATCCTGCTGCTGCTGTGTCG & CTTCGTTTAGCGTGAAGCAACG \\
\hline 176 & RM585 & 6 & 3.16 & AG & 45 & CTAGCTAGCCATGCTCTCGTACC & CTGTGACTGACTTGGTCATAGGG \\
\hline 177 & RM204 & 6 & 3.17 & CT & 44 & GTGACTGACTTGGTCATAGGG & GCTAGCCATGCTCTCGTACC \\
\hline 178 & RM217 & 6 & 4.23 & CT & 20 & ATCGCAGCAATGCCTCGT & GGGTGTGAACAAAGACAC \\
\hline 179 & RM314 & 6 & 4.84 & $\begin{array}{c}\text { GT,CG } \\
\text {,GT }\end{array}$ & $8,3,5$ & CTAGCAGGAACTCCTTTCAGG & AACATTCCACACACACACGC \\
\hline 180 & RM253 & 6 & 5.44 & AG & 22 & CCATCTCTGCCTCTGACTCACC & TCCTTCAATGGTCGTATCTTCTCC \\
\hline 181 & RM19771 & 6 & 9.04 & AT & 47 & AACCAATGCACACTTCTTCTGTGC & CAACTGTAGAGGTTGGAATGATCTGC \\
\hline 182 & RM8226 & 6 & 9.32 & AAG & 14 & GCGTGTTCAGAAATTAGGATACGG & GATCTCGCCACGTAATTGTTGC \\
\hline 183 & RM564 & 6 & 11.61 & AC & 14 & TCCTTCTGCATCAATTCCTCTCG & CTCCATAGCCTTGTTAAGTGATGAGC \\
\hline 184 & RM20185 & 6 & 20.31 & AT & 44 & AAACCGCTCAACTTTAGCTAGG & ACCTCTACTCGTTTGTGTCTGG \\
\hline 185 & RM20242 & 6 & 20.97 & AG & 23 & CTAGCCAAACTCACTTGTCATCC & GATATGACTGATTGGGACAGTGG \\
\hline 186 & RM162 & 6 & 23.65 & AC & 20 & TTGTTCCAGTTCAGGTCTTGTGC & CCCTACAAACACCATAAGAAGCAACC \\
\hline 187 & RM20476 & 6 & 25.76 & AAT & 18 & ATGGACTGAGAACCCAAACAACG & GCTGTGTGTATTCTTGGTTGATGC \\
\hline 188 & RM528 & 6 & 26.17 & AGAT & 9 & GGAGGAGAACCATATTTGGCAAGG & AGCTTGGACCACTTTCAACACTAAGC \\
\hline 189 & RM30 & 6 & 26.87 & AG & 13 & GTCACATTCCGTTTCCCATCATTCC & СCTCACCTCACCACACGACACG \\
\hline 190 & RM400 & 6 & 28.05 & AAT & 63 & TTACACCAGGCTACCCAAACTCG & TTGCTGAGTTCCCTCGTCTATCC \\
\hline 191 & RM340 & 6 & 28.22 & AAG & 10 & GGGTAAATGGACAATCCTATGG & ACCCTATTCTGGAGTTCATCTGG \\
\hline 192 & RM20632 & 6 & 28.22 & AAG & 10 & GGGTAAATGGACAATCCTATGG & ACCCTATTCTGGAGTTCATCTGG \\
\hline 193 & RM20633 & 6 & 28.23 & AG & 10 & ТТАСССТССТССТССТССТТСТАТСС & TCAACCACAGTGCAGTGACTTCG \\
\hline 194 & RM439 & 6 & 29.11 & AAT & 13 & CTGGGTCTAATCTCGTCCTAAATTGC & CGCCTCTCATAACAGTCCACTCC \\
\hline 195 & RM176 & 6 & 29.75 & CCG & 7 & TACGACGTCTCCAGCCTCATCC & CGCTAATCAATCAGAGCGATGC \\
\hline 196 & RM20779 & 7 & 0.10 & AT & 31 & TGTCTTCTCCTAGGTTGTGTGATCG & TCTTGGCTCTACATGCTCCTTACG \\
\hline 197 & RM20834 & 7 & 0.59 & AC & 43 & GATATGGTTCCACTTCACCATGC & TTAGAAACTCGCCTTCAGAACTGC \\
\hline 198 & RM481 & 7 & 2.88 & CAA & 12 & TAGCTAGCCGATTGAATGGC & СTCСACCTCCTATGTTGTTG \\
\hline 199 & RM5499 & 7 & 10.02 & AG & 25 & GGACGAAAGGGTATTTGATTGG & CCTCAAGGTGGTCTCCTTCTCC \\
\hline 200 & RM542 & 7 & 12.66 & AG & 22 & CGCTCCTTTCTAGCTCCATCTCC & AACTGCAACGAGTAAGGCAGAGG \\
\hline 201 & RM320 & 7 & 18.64 & AT & 18 & TACAGCAGGGTGGAATATCGACAAGC & TCGTTGTAACGCGTGGATATTTGG \\
\hline 202 & RM560 & 7 & 19.53 & AG & 12 & ATTTGGATGTCATCGCCAAGG & CCTGATTCGGTGAACACATAAAGC \\
\hline 203 & RM346 & 7 & 21.05 & $\mathrm{CTT}$ & 18 & CGAGAGAGCCCATAACTACG & ACAAGACGACGAGGAGGGAC \\
\hline 204 & RM336 & 7 & 21.82 & AAG & 18 & GTATCTTACAGAGAAACGGCATCG & GGTTTGTTTCAGGTTCGTCTATCC \\
\hline 205 & RM10 & 7 & 22.19 & GA & 15 & TTGTCAAGAGGAGGCATCG & CAGAATGGGAAATGGGTCC \\
\hline 206 & RM70 & 7 & 22.78 & ATT & 33 & GTGGACTTCATTTCAACTCG & GATGTATAAGATAGTCCC \\
\hline 207 & RM234 & 7 & 25.42 & AG & 13 & TTCAGCCAAGAACAGAACAGTGG & СTTCTCTTCATCCTCCTCCTTGG \\
\hline 208 & RM5455 & 7 & 26.41 & AG & 18 & GATCAACGAACCCACCACACC & CGCGTCTTGTATATGCACTTGATCC \\
\hline 209 & RM172 & 7 & 29.56 & AGG & 6 & TGCAGCTGCGCCACAGCCATAG & CAACCACGACACCGCCGTGTTG \\
\hline 210 & RM5911 & 8 & 0.07 & ATT & 22 & СCCTCTTTTTAAGTCTGGGG & GGTGCCTCCTTTCAAAGTTG \\
\hline 211 & RM6369 & 8 & 0.12 & AAG & 14 & AGCTAGCTTCACCTACCTACCTCACC & ATGGTCATGTCGTTGGTTTGC \\
\hline 212 & RM22212 & 8 & 0.18 & AGC & 8 & TCCTCTGAATCTTCACAGTTGG & GAAGAAGAACTCGAAGCATGG \\
\hline 213 & RM1019 & 8 & 0.2 & AC & 13 & AGGACTTGTCATAACCTCCAAGC & AATGCAAGTATCCACCTCTTGTCC \\
\hline
\end{tabular}

Journal of Experimental Biology and Agricultural Sciences http://www.jebas.org 


\begin{tabular}{|c|c|c|c|c|c|c|c|}
\hline S. N & $\begin{array}{c}\text { Name of } \\
\text { SSR } \\
\text { marker }\end{array}$ & $\begin{array}{l}\text { Chr. } \\
\text { No }\end{array}$ & $\begin{array}{c}\text { Physical } \\
\text { position } \\
(\mathrm{Mb})\end{array}$ & Motif & $\begin{array}{l}\text { Number } \\
\text { of } \\
\text { Repeats }\end{array}$ & Forward Primer Sequence & Reverse Primer Sequence \\
\hline 214 & RM310 & 8 & 5.11 & GT & 19 & CCAAAACATTTAAAATATCATG & GCTTGTTGGTCATTACCATTC \\
\hline 215 & RM547 & 8 & 5.59 & ATT & 19 & TAGGTTGGCAGACCTTTTCG & GTCAAGATCATCCTCGTAGCG \\
\hline 216 & RM22565 & 8 & 5.79 & ACAT & 15 & TCCACGCGTTGTCGTAGAAATTTAGC & AGCCCGAGCACCATGAAACACC \\
\hline 217 & RM22679 & 8 & 8.57 & AG & 22 & CCTCATCATCGTCTCCGACTGC & TCTCAAACCCTCAACAATGTCTCG \\
\hline 218 & RM22685 & 8 & 8.8 & AGG & 10 & ATGGGCTTCCAGGCTCAATCTCG & СCСАCTCTCACGTCTCCTCTCTTCC \\
\hline 219 & RM22688 & 8 & 8.85 & AAT & 28 & CTTCGATCTCTTGCCTGGATACC & CCATTTCGTTGGGTTTCAGTCC \\
\hline 220 & RM22695 & 8 & 8.98 & AAT & 16 & CAAGGGATTCACTAACGGTTCCTTCC & CACATTGAACCGTTGCTTTGTGC \\
\hline 221 & RM483 & 8 & 11.92 & AT & 26 & CTTCCACCATAAAACCGGAG & ACACCGGTGATCTTGTAGCC \\
\hline 222 & RM22865 & 8 & 13.74 & AT & 32 & NA & NA \\
\hline 223 & RM404 & 8 & 15.43 & AG & 29 & GGAGCAGCTAAGGCAGATAAGAGG & GCCTTCATGCTTCAGAAGACAGC \\
\hline 224 & RM339 & 8 & 17.94 & $\begin{array}{l}\text { CTT, } \\
\text { CCT, } \\
\text { CTT }\end{array}$ & $8,1,5$ & GTAATCGATGCTGTGGGAAG & GAGTCATGTGATAGCCGATATG \\
\hline 225 & RM515 & 8 & 20.28 & AG & 11 & CGAGATACGGTGAATGACACACG & ACCAATCAGAGAGCACACACAGG \\
\hline 226 & RM223 & 8 & 20.65 & AG & 20 & GCTGGGCCTATATGGACTTTCG & AGGCAAGTCTTGGCACTGTAAGC \\
\hline 227 & RM284 & 8 & 21.14 & AG & 12 & ACTGCATGATCCTCCTCAGATCC & СССTCTGATCTCTGATACTCCATCC \\
\hline 228 & RM256 & 8 & 24.27 & CT & 21 & GACAGGGAGTGATTGAAGGC & GTTGATTTCGCCAAGGGC \\
\hline 229 & RM80 & 8 & 24.47 & TCT & 25 & TTGAAGGCGCTGAAGGAG & CATCAACCTCGTCTTCACCG \\
\hline 230 & RM23408 & 8 & 25.08 & AT & 47 & ССАTCTCAАCTCCTTCGTTTACTGC & TCGACTGTTTGCTTGGAATAGGC \\
\hline 231 & RM419 & 8 & 25.82 & AG & 12 & TTCCTTCCCAGTGATTTCTCTCC & CCCTTTCTCCTCCTTTGGTATGC \\
\hline 232 & RM458 & 8 & 27.35 & TAG & 8 & GGTGATCTGCATTGTCAACG & TGCAATGGATCTAGCGACTG \\
\hline 233 & RM281 & 8 & 27.89 & GA & 21 & ACCAAGCATCCAGTGACCAG & GTTCTTCATACAGTCCACATG \\
\hline 234 & RM23668 & 9 & 0.60 & ACG & 10 & TGCATAGCATATCAACTAGCCCTACC & GCTGAAACAGAATGAAAGCACAGC \\
\hline 235 & RM23679 & 9 & 0.82 & AAAG & 10 & TCACAGCTTAGTGCATGTTGAGC & GATTCACCTGGCAATGAGAACG \\
\hline 236 & RM316 & 9 & 1.02 & $\begin{array}{c}\text { GT,TG, } \\
\text { TTTG, } \\
\text { TG }\end{array}$ & $8-9,4,4$ & CTAGTTGGGCATACGATGGC & ACGCTTATATGTTACGTCAAC \\
\hline 237 & RM6196 & 9 & 5.4 & CCG & 8 & GGGCACGGACTACCAAAGGAAGC & ATCAGGCCCAGCACCCAAACC \\
\hline 238 & RM23914 & 9 & 7.19 & AT & 23 & GAGGATCCTTACCATCAAACTTCG & CCAAGAACCTGCATTCTTCAAGG \\
\hline 239 & RM7390 & 9 & 10.42 & AGAT & 20 & TGAGAGCTCGTAGGAAGTGTCC & CAGAGTCAGCAATCGCTAAGG \\
\hline 240 & RM3912 & 9 & 10.77 & AC & 22 & CACTCAGATTTGGCCGATCC & GCTGATCCAGATCTACCTGACACC \\
\hline 241 & RM24166 & 9 & 12.34 & AGCG & 5 & CTCTTCGCTGACCTCCTGTGG & GTCGGTGGTGTACATCTGCTTCG \\
\hline 242 & RM24188 & 9 & 12.59 & $\mathrm{AC}$ & 18 & GAAAGCGACACCTGATAGATACAGC & CAAACTGGATGTCAGTTGTGATGG \\
\hline 243 & RM24263 & 9 & 14.13 & AT & 30 & CTCATCGGCGACATATCACAGC & ATGGGATGTACACAGCCAAACG \\
\hline 244 & RM3700 & 9 & 15.37 & AG & 15 & СCTTTGCCGCCTTCTCTTGG & ACGAGTTCCCGGTTAACCTTACG \\
\hline 245 & RM24353 & 9 & 15.45 & AG & 31 & GTTCTTCCCTACTGTGAACATGG & GTGAAATATCCCTATGCTCTCACC \\
\hline 246 & RM24355 & 9 & 15.49 & AG & 11 & CGCCAAGGACAATCCAAGAACG & GTGTGTAATGCGTGTGAAACTGATGG \\
\hline 247 & RM24357 & 9 & 15.5 & CCG & 7 & ACGCTTGCCTTCTCATCGTCTCG & CTGGAACGTCGCCACGTACTGC \\
\hline 248 & RM160 & 9 & 19.49 & AAG & 23 & CCCAAATCAGGAAAGTTTCTCAGC & AGTCATCCTTGGCTACCAGATGC \\
\hline 249 & RM24856 & 10 & 0.10 & ACGG & 5 & CCTTCACGTGTGTTATCACGTTCC & AGAAATGGACAAGAGCATGGTAGC \\
\hline 250 & RM24875 & 10 & 0.28 & ACAT & 11 & GATGAGCGAGGACAAGAATTAAGC & GCTAGCTGATTCAGATCCCATCG \\
\hline
\end{tabular}

Journal of Experimental Biology and Agricultural Sciences http://www.jebas.org 


\begin{tabular}{|c|c|c|c|c|c|c|c|}
\hline S. N & $\begin{array}{l}\text { Name of } \\
\text { SSR } \\
\text { marker }\end{array}$ & $\begin{array}{l}\text { Chr. } \\
\text { No }\end{array}$ & $\begin{array}{c}\text { Physical } \\
\text { position } \\
(\mathrm{Mb})\end{array}$ & Motif & $\begin{array}{c}\text { Number } \\
\text { of } \\
\text { Repeats }\end{array}$ & Forward Primer Sequence & Reverse Primer Sequence \\
\hline 251 & RM474 & 10 & 1.8 & AT & 13 & TACACGAGGGAGTACTCGAATGG & CATGGAGGTATAGAAGAGCATTGG \\
\hline 252 & RM216 & 10 & 4.99 & AG & 12 & GATGGTAAAGGAAGAACGTGTGC & CACTCATAGACGCATCACATAGCC \\
\hline 253 & RM467 & 10 & 13.04 & AG & 21 & TGTTGTCACATGAGATGGCTATGC & GCTGACCTTGTGAGACGTTTAGACC \\
\hline 254 & RM258 & 10 & 17.57 & AG & 11 & CTCCCTGGCCTTTAAAGCTGTCG & GACGAACAGCAGCAGAAGAGAAGC \\
\hline 255 & RM6100 & 10 & 18.37 & ACG & 8 & TTCCCTGCAAGATTCTAGCTACACC & TGTTCGTCGACCAAGAACTCAGG \\
\hline 256 & RM25735 & 10 & 19.56 & AAG & 25 & AGGCAGGCAAGCAGTAGTTTCG & ATCAAGATCAGGAGCCGCAAGG \\
\hline 257 & $\mathrm{RM} 25745$ & 10 & 19.68 & AT & 11 & TGGAGATGAGTTCCCAGCTACTTCC & CGTGTGTCTTCCTGTTTGGTATGG \\
\hline 258 & RM25766 & 10 & 20.1 & AG & 15 & CTGGGTAGTTGTTTCTCATGTGC & AGTGCTATTGCCATTGTACTGC \\
\hline 259 & RM5352 & 10 & 20.67 & AG & 13 & CATGAAGAGGAGATCAATGTAGCC & CTAAACATGGTGCAAGTGATGG \\
\hline 260 & RM496 & 10 & 21.98 & AG & 24 & GACATGCGAACAACGACATCC & CTATAGTTGTTGCACATGCGATCC \\
\hline 261 & RM25893 & 10 & 22.11 & AAG & 7 & GAAGAAGATGGCGATGAAACTTGG & CAGAGCGGAGATCCAGATTGACC \\
\hline 262 & RM25895 & 10 & 22.14 & ACG & 7 & CTCTGTCGTCCACTCCAGATTCC & GTCGAATTGAGAGGGATTGATGG \\
\hline 263 & RM25898 & 10 & 22.18 & AT & 30 & GCAGCCAATCAGAAATCATAGC & AAATATGTCCGTGTCCCAATGC \\
\hline 264 & RM26018 & 11 & 1.22 & AT & 16 & GGCGAGGACACCAGTAGACAGC & GCCAGCCACTAGATCCACATGC \\
\hline 265 & RM26092 & 11 & 2.69 & ATC & 7 & GTCATCGTCGTGGTCGCAGTCC & TCTGGAGTGGCGTTGACTGTTGC \\
\hline 266 & RM26097 & 11 & 2.74 & AGG & 7 & ATGGTTCAAATGTGGAGGAGAGG & GAACCTTCCAGAACAATCCAGTATCC \\
\hline 267 & RM26105 & 11 & 2.83 & AG & 10 & AGCATCAATTCAGCTTGCTTGC & TTCTGGTTCTTTGAGAGAGTGTGC \\
\hline 268 & RM26168 & 11 & 3.91 & CCG & 7 & AGGTTGCGCCGCTTGTACACC & CTTCGTCCACGACCACGAGAGC \\
\hline 269 & RM167 & 11 & 4.06 & AG & 19 & CTCCGAGTCCGACCACAAGG & TCCAGCCCTTCCTATCATATTGC \\
\hline 270 & RM26185 & 11 & 4.27 & AGAT & 5 & CTGAGAATTTCTATCCTCCGATGC & GCAAATTATGGCAGCAAGATCC \\
\hline 271 & RM552 & 11 & 4.82 & AAT & 13 & CGCAGTTGTGGATTTCAGTGC & TCATGCTCAACGTTTGACTGTCC \\
\hline 272 & RM202 & 11 & 8.91 & AG & 24 & TGGAACACCCATAGACAAACAGC & TGGCAAGTGGTATTCTTCCTTCC \\
\hline 273 & RM26463 & 11 & 10.13 & AT & 38 & CGGATTTCCGAATCGTTCAGAGC & AACCCTGCAACCAAAGCCATAGG \\
\hline 274 & RM287 & 11 & 16.61 & AG & 15 & GGCTACACCTACACGCGAGAACC & AGATGCATGGAATGCCTGTTTGG \\
\hline 275 & RM209 & 11 & 17.8 & $\mathrm{CT}$ & 18 & ATATGAGTTGCTGTCGTGCG & CAACTTGCATCCTCCCCTCC \\
\hline 276 & RM206 & 11 & 21.63 & AG & 33 & ATCGATCCGTATGGGTTCTAGC & GTCCATGTAGCCAATCTTATGTGG \\
\hline 277 & RM254 & 11 & 24.23 & $\begin{array}{c}\text { TC,AT } \\
\text { T,CT }\end{array}$ & $6,1,11$ & AGCCCCGAATAAATCCACCT & CTGGAGGAGCATTTGGTAGC \\
\hline 278 & RM27147 & 11 & 24.26 & AT & 14 & CACTGGCAAAGTCAGTAGTCCTTCC & GGAGGCTAGCTCCGTAAATCTGG \\
\hline 279 & RM27150 & 11 & 24.32 & AG & 10 & ATTCAGGCTCGCTTACCATCTCC & CCTCTGCTTGTCCCAAATCACC \\
\hline 280 & RM224 & 11 & 26.8 & AAG,AG & 8,13 & ATCGATCGATCTTCACGAGG & TGCTATAAAAGGCATTCGGG \\
\hline 281 & RM27336 & 11 & 27.76 & AT & 19 & AGCAGTGATTCATCGCTCTATCG & GTGATGCTGTGCCGATAATATTCC \\
\hline 282 & RM5926 & 11 & 28.33 & AAT & 30 & TAGGTCCATCCAAATCTCGATCC & TGGCAGAGGAGATTAGAGTAATACGG \\
\hline 283 & RM5367 & 12 & 0.17 & AG & 13 & ATTCCTCACGCCCACTGTACC & AGTGATATCCAACCCGAACTTGC \\
\hline 284 & RM6371 & 12 & 1.08 & AAG & 14 & ACGACCACCGTGTACGAAAGC & GATGAAGACGGATGAGGTGTTGC \\
\hline 285 & RM19 & 12 & 2.43 & ATC & 10 & CAAAAACAGAGCAGATGAC & CTCAAGATGGACGCCAAGA \\
\hline 286 & RM247 & 12 & 3.19 & CT & 16 & TAGTGCCGATCGATGTAACG & CATATGGTTTTGACAAAGCG \\
\hline 287 & RM491 & 12 & 3.58 & AT & 14 & CACATGATGCGTAGCGAGTTGC & TTATGCCTCTCCCTTCCCAATTCC \\
\hline 288 & RM511 & 12 & 17.4 & ACG & 7 & AACGAAAGCGAAGCTGTCTCC & ATTTGTTCCCTTCCTTCGATCC \\
\hline 289 & RM1261 & 12 & 17.58 & AG & 16 & ATGGTAGAGACACAAGTCCATGC & GACAAATTGGTGTAGGTGAAGG \\
\hline
\end{tabular}

Journal of Experimental Biology and Agricultural Sciences http://www.jebas.org 


\begin{tabular}{|cccccccc|}
\hline S. N & $\begin{array}{c}\text { Name of } \\
\text { SSR } \\
\text { marker }\end{array}$ & $\begin{array}{c}\text { Chr. } \\
\text { No }\end{array}$ & $\begin{array}{c}\text { Physical } \\
\text { position } \\
(\mathrm{Mb})\end{array}$ & Motif & $\begin{array}{c}\text { Number } \\
\text { of } \\
\text { Repeats }\end{array}$ & Forward Primer Sequence & Reverse Primer Sequence \\
\hline 290 & RM28166 & 12 & 17.65 & AG & 12 & TGCTTGCAAACATTGCTTCTGG & ACTGATGTACTGAACACGGGAAGG \\
\hline 291 & RM519 & 12 & 19.97 & AAG & 8 & AATTTCCGCGAAATCAGCATCC & TCATCTGGACAGTCGAGGTACGC \\
\hline 292 & RM313 & 12 & 20.87 & $\begin{array}{c}\text { GT,CA }, \\
\text { CG,GT }\end{array}$ & $6,1,5-6,8$ & TGCTACAAGTGTTCTTCAGGAC & GCTCACCTTTTGTGTTCCAC \\
\hline 293 & RM309 & 12 & 21.52 & AC & 14 & CACGCACCTTTCTGGCTTTCAGC & AGCAACCTCCGACGGGAGAAGG \\
\hline
\end{tabular}

background of the two selected parents. The selection of genotypically diverse parents is imperative for any QTL mapping program. The geographical diverse origin of parents reflects in genotypic variations in terms of polymorphism. Mutual relation between the geographic distribution of germplasm and polymorphism of molecular markers has been also noticed by $\mathrm{He}$ et al. (2004). Also, a significant correlation between genetic relationship and geographical place of indica local rice varieties has been indicated (Zhang, 2005).

As SSR based polymorphism is characterized by repeat motifs (di, tri, tetra); in the current study also it was observed that SSR repeat motifs with dinucleotide repeats were more polymorphic than others. Out of 294 polymorphic markers obtained from screening 1013 SSRs, 1 marker possesses a single nucleotide repeat, 182 contained dinucleotide repeats, 87 showed trinucleotide repeats and 23 were having tetranucleotide repeats. Out of the 182 , dinucleotide repeats AG motif appeared more polymorphic than others, which constitutes $49.45 \%$ of dinucleotide repeats, followed by AT repeats $(21.97 \%)$. The abundance of $(\mathrm{AG})_{\mathrm{n}}$ and $(\mathrm{AT})_{\mathrm{n}}$ dinucleotide repeat has been noticed by earlier studies (Grover et al., 2007; Kumar et al., 2013). Out of 87 trinucleotides, AAG repeated 23 times representing $26.43 \%$ of its total, followed by AAT 12 times (13.79\%). It was also observed that GC rich trinucleotide repeats viz., ACG, AGC, CGG, CCG are at a variable frequency (Figure 2). The occurrence of $80 \%$ trinucleotide GC rich repeats in predicted exons and their frequent association with genes (or ESTs) and (AC)n/(AT)n dinucleotides and tetranucleotide repeat motif in the non-coding intergenomic region has been reported (Temnykh et al., 2001). Out of 23 tetranucleotide repeats, AGAT appeared 6 times (26.08\%). In a comparative study across the genome of two subspecies of Oryza sativa viz ssp. indica and ssp. japonica abundance of (AGAT) repeats has been observed (Grover et al., 2007).

In the present study tetra nucleotides were found to be frequent for chromosomes 5 and 9 and least frequent in chromosome 8, 11, 12, and 4 . Tri nucleotides were found to be highest for chromosome 4 and less frequent in chromosomes 9, 12, and 7 (Figure.3). Similarly long trinucleotides repeats have been reported on chromosome 1, 4, and 12 (Grover et al., 2007). Dinucleotides were most frequent for chromosome 1 and less frequent in chromosome 12 and 9 . The richness of $(\mathrm{AT})_{\mathrm{n}}$ dinucleotide repeat motif has been explained by the presence of micron transposable sequences that particularly target $(\mathrm{AT})_{\mathrm{n}}$ repeats (Temnykh et al., 2001; Akagi et al., 2001; Grover et al., 2007). Out of 840 hypervariable rice microsatellite (hvRM) markers identified, markers with dinucleotides repeat motifs were more in number followed by markers with tri and tetranucleotides repeat motifs (Narshimulu et al., 2011). In present study number of repeats ranged from 5 to 63 . Details of repeat motif, length of repeats, and physical position for all the polymorphic microsatellites in the present study will assist in selection for set of polymorphic microsatellites to be used in genotyping of mapping population for QTL mapping and markerassisted selection in breeding.

Distribution of polymorphic markers based on their physical position (Gramene database) is almost uniform for chromosome 2, 3 , and 9 (gap between polymorphic markers is less than $5 \mathrm{Mb}$ ) but in chromosome 12, 10, 6, 1, and 7 longer stretches of DNA found to be monomorphic indicating conserved sequences distributed in the two parents selected for the study. The importance of evenly distributed markers for analysis and to generate few but highly informative data points either for mapping or threshold recovery of a recurrent parent in marker assisted backcross breeding has been highlighted (Prigge et al, 2009; Servin \& Hospital, 2002). Nonrandom and non-uniform distribution of microsatellite markers in plant genome have also been reported (Morgante et al., 2002). Also, preferential positioning of the microsatellite in the rice genome was revealed (Grover et al., 2007). The reason behind this kind of positioning of markers has been explained as DNA replication and repair mechanisms during the synthesis of new repeats and elimination of existing repeats (Schlotterer, 2000). Low polymorphic regions can be recovered by screening more SSR markers for a given region based on available resources and published literature on the genome map of rice.

The identified polymorphic markers from this investigation will be utilized for the mapping of major QTLs/ Candidate genes associated with different micro-nutrients and quality-related traits 


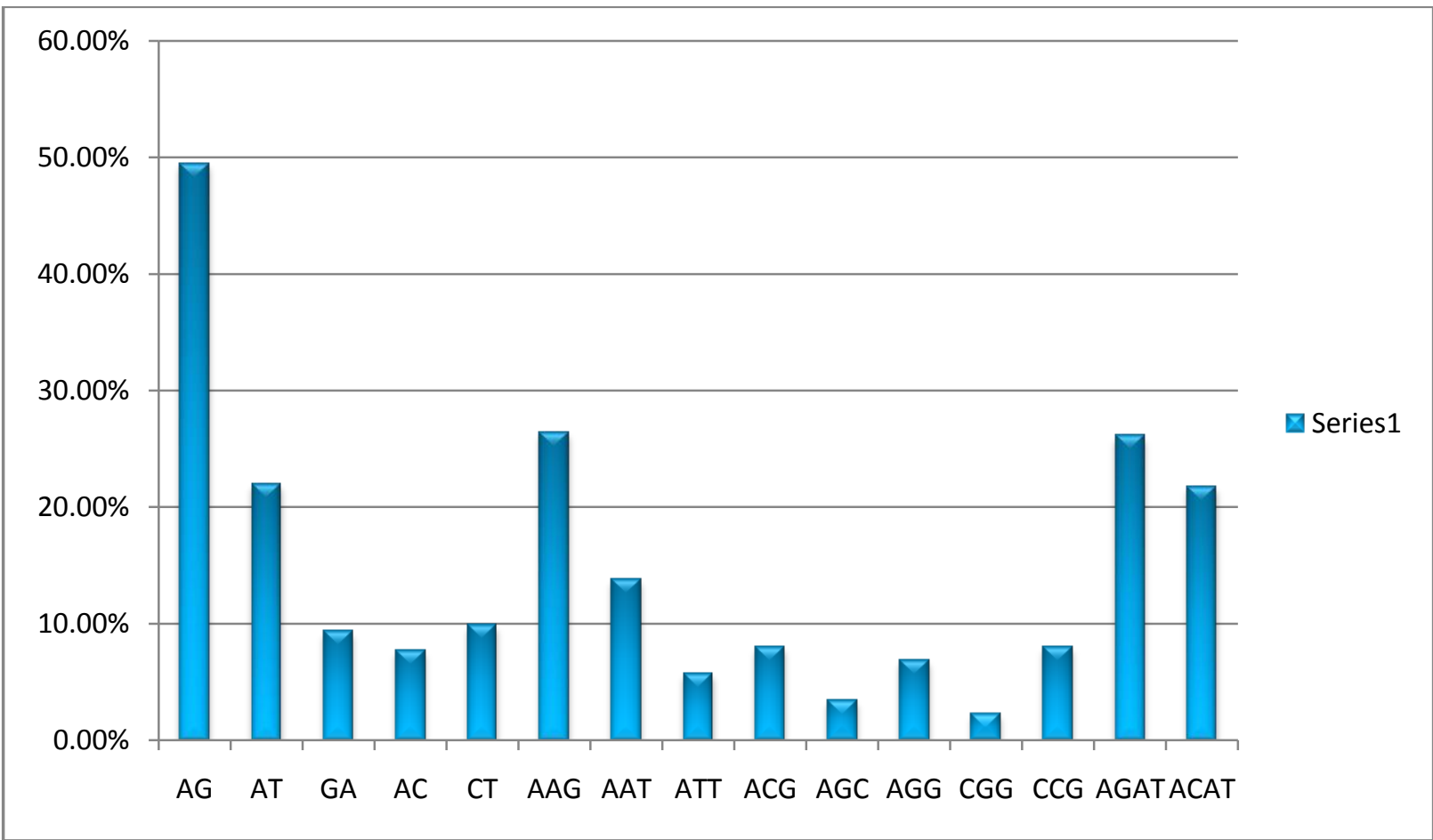

Figure 2 Frequency distribution of different types of SSR repeat motifs (di,tri, tetra)

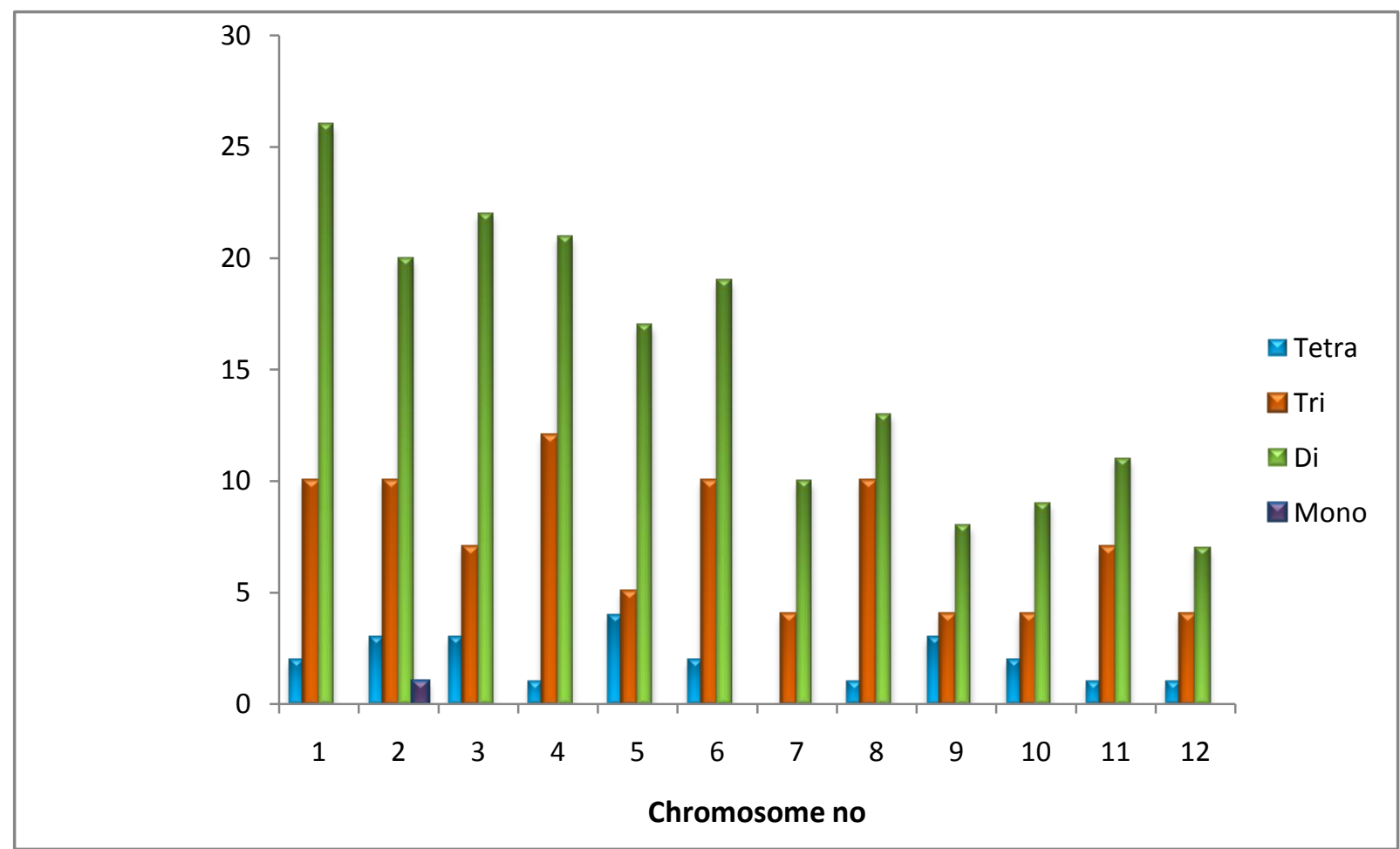

Figure 3 Number of different types of repeat motifs across the 12 chromosomes of Rice 

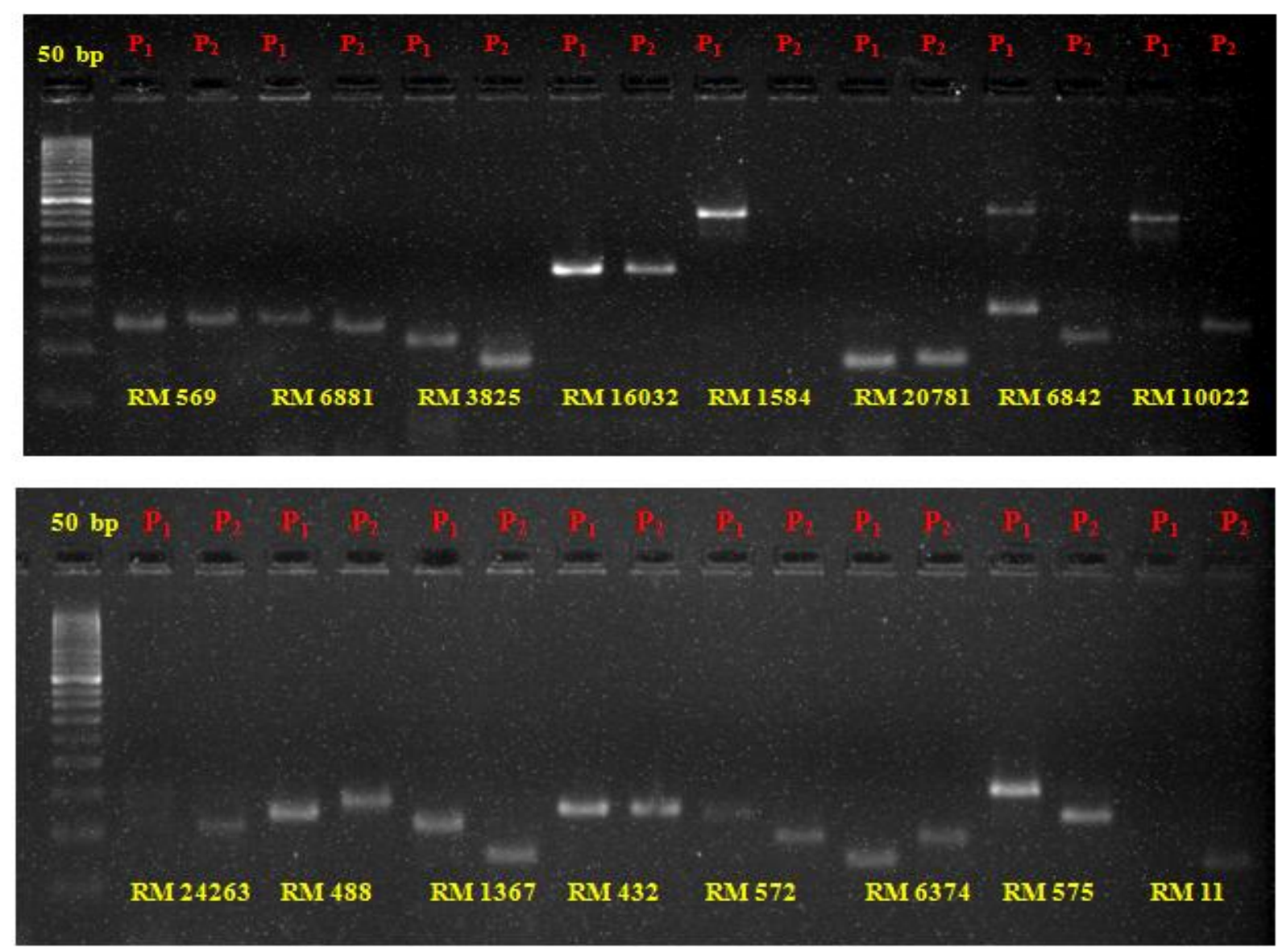

Figure 4 Parental polymorphism profile of parents; Rajendrakasturi $\left(\mathrm{P}_{1}\right)$ and URG-30 $\left(\mathrm{P}_{2}\right)$; here 50bp- DNA ladder, P1-Parent 1 (Rajendrakasturi), P2-Parent 2 (URG-30)

in rice. Once identified QTL region defined by marker interval can be narrowed down further by fine-mapping approaches. In detail scanning of genome-wide polymorphic SSR resources for a given biparental mapping population, provides way forward for studying 'Causal polymorphism in terms of SNP variant' in a genomic region defined by QTL interval (Gupta et al., 2019). Recent advancement in sequencing technologies has strengthened this possibility by highthroughput genotyping and NGS-led QTL-seq assay. QTL-seq analysis and differential expression profiling studies were performed by Daware et al. (2016) to identify a potential candidate and allelic variants for grain weight trait in rice is an example of successful integrated use of SSR and SNP markers data.

\section{Conclusion}

Breeding mineral dense rice variety is one of the sustainable approaches for ensuring the nutritional security of the nation. Local genotypes and landraces are a proven source of higher mineral content and the present study attempts to make use of local genotype to identify new genomic regions for high grain zinc and iron content. Identified markers showing allelic variant for whole genome wide SSRs covering the whole genome of rice in the present parental polymorphism study will be utilized for genotyping of whole mapping population developed from the above cross and better understanding of mapping QTLs/genes for high grain zinc, iron, grain quality, and yield traits. It can significantly contribute towards the development of mineral dense rice variety using marker assisted selection and marker assisted backcross approaches.

\section{Acknowledgement}

The authors gratefully acknowledge the financial support provided by Department of Science and Technology (DST), Ministry of Science and Technology, Government of India, through DST INSPIRE fellowship (IF170506) to the first author for pursuing fulltime doctoral (Ph. D.) degree programme at Banaras Hindu University, Varanasi, as well as thankful to Harvest Plus project on Developing high zinc rice for Eastern India (IFPRI, Washington D.C. and CIAT, Columbia) for providing funds for conducting the 
trial and thankful to Dr. Arvind Kumar, Director ISARC, Varanasi and Dr. Vikas Kumar Singh, Coordinator, IRRI-SAH, Hyderabad for providing resources and facilities to carry out above research work.

\section{Conflicts of interest}

The authors declare that there is no conflict of interest.

\section{References}

Akagi H, Yokozeki Y, Inagaki A, Mori K, Fujimura T (2001) Micron, a microsatellite-targetting transposable element in the rice genome. Molecular Genetics and Genomics 266(3): 471-480.

Bollinedi H, Yadav AK, Vinod KK, Krishnan SG, Bhowmick PK, Nagarajan M, Neeraja CN, Ellur RK, Singh AK (2020) GenomeWide Association Study Reveals Novel Marker-Trait Associations (MTAs) Governing the Localization of $\mathrm{Fe}$ and $\mathrm{Zn}$ in the Rice Grain. Frontiers in genetics, 11. doi.org/10.3389/fgene.2020.00213

Bouis HE, Saltzman A (2017) Improving nutrition through biofortification: a review of evidence from HarvestPlus, 2003 through 2016. Global Food Security 12: 49-58.

Bouis HE, Welch RM (2010) Biofortification-a sustainable agricultural strategy for reducing micronutrient malnutrition in the global south. Crop Science 50:S20-S32.

Challa V, Kole PC (2019) Parental evaluation and polymorphism survey of drought contrasting donor and recurrent parents in rice (Oryza sativa L) using microsatellite markers. Electronic Journal of Plant Breeding 10(2): 406-412.

Cho YG, Ishii T, Temnykh S, Chen X, Lipovich L, McCouch SR Park WD, Ayres N, Cartinhour S (2000) Diversity of microsatellites derived from genomic libraries and GenBank sequences in rice (Oryza sativa L.). Theoretical and Applied Genetics 100: 713-722.

Das P, Adak S, Majumder AL (2020) Genetic manipulation for improved nutritional quality in rice. Frontiers in Genetics 11:776.doi.org/10.3389/fgene.2020.00776

Daware A, Das S, Srivastava R, Badoni S, Singh AK, Agarwal P, Parida SK, Tyagi AK (2016) An efficient strategy combining SSR markers-and advanced QTL-seq-driven QTL mapping unravels candidate genes regulating grain weight in rice. Frontiers in plant science 7: 1535. 16. doi.org/10.3389/fpls.2016.01535

Gautam RK, Srikumar M, Singh PK, Singh AK, Sakthivel K, Ahmad I, Devakumar K (2015) Polymorphism Between Popular Rice Varieties of Andaman and Bacterial Blight Resistant Donor IRBB60 Revealed Through Highly Variable SSR Markers. Vegetos (International Journal of Plant Research) 29: 1-5
Ghose B, Sarker S, Kpoghomou MA, Gao H, Jun L, Yin D, Ghosh S (2013) Self sufficiency in rice and food security: a South Asian perspective. Agriculture \& Food Security 2(1): 10

Gramene marker database (2019) Gramene marker database available at https://www.gramene.org/ accessed on 20 July, 2019.

Grover A, Aishwarya V, Sharma PC (2007) Biased distribution of microsatellite motifs in the rice genome. Molecular Genetics and Genomics 277(5): 469-480.

Gupta PK, Kulwal PL, Jaiswal V (2019) Association mapping in plants in the post-GWAS genomics era. In Advances in genetics, Academic Press. (Vol. 104, pp. 75-154).

HarvestPlus (2014) Biofortification progress briefs available on http://www.harvestplus.org/sites

/default/files/Biofortification_Progress_Briefs_August2014_WEB

_0.pdf accessed on $10^{\text {th }}$ July, 2020.

He HQ, Jia XL, Liang YY, Shen LH, Song BQ, Guo YC, Liang KJ, Lin WX (2004) Assessment of genetic diversity of allelopathic rice germplasm based on RAPD and ISSR. Acta Genetica Sinica 31(9): 888-894.

Hotz C, Brown KH (2004) Assessment of the risk of zinc deficiency in populations and options for its control. Food and Nutrition Bulletin 25: S91-S204.

Kim SR, Yang J, An G, Jena KK (2016) A simple DNA preparation method for high quality polymerase chain reaction in rice. Plant Breeding and Biotechnology 4:99-106.

Kumar GS, Kumari KA, Rani CVD, Sundaram RM, Vanisree S, Jamaloddin, M, Swathi G (2013) Study of simple sequence repeat (SSR) polymorphism for biotic stress resistance in elite rice variety JGL 1798. African Journal of Biotechnology 12(40): 5833-5840.

Lee SM, Kang JW, Lee JY, Seo J, Shin D, Cho JH, Jo S, Song YC, Park DS, Ko JM, Koh HJ, Lee JH (2020) QTL Analysis for Fe and Zn Concentrations in Rice Grains Using a Doubled Haploid Population Derived from a Cross Between Rice (Oryza sativa) Cultivar 93-11 and Milyang 352. Plant Breeding and Biotechnology 8(1): 69-76.

McCouch SR, Teytelman L, Xu Y, Lobos KB, Clare K, Walton M, Fu B, Maghirang R, Li Z, Xing Y, Zhang Q, Kono I, Yano M, Fjellstrom R, Declerck G, Schneider D, Cartinhour S, Ware D, Stein L (2002) Development and mapping of 2240 new SSR markers for rice (Oryza sativa L.) (supplement). DNA Research : an International Journal for Rapid Publication of Reports on Genes and Genomes 9: 257-279.

Morgante M, Hanafey M, Powell W (2002) Microsatellites are preferentially associated with nonrepetitive DNA in plant genomes. Nature Genetics 30(2):194-200. 
Narshimulu G, Jamaloddin M, Vemireddy LR, Anuradha G, Siddiq E (2011) Potentiality of evenly distributed hypervariable microsatellite markers in marker- assisted breeding of rice. Plant Breeding 130(3): 314-320.

Pradhan SK, Pandit E, Pawar S, Naveenkumar R, Barik SR, Mohanty SP, Nayak DK, Ghritlahre SK, Rao DS, Reddy JN, Patnaik SSC (2020b) Linkage disequilibrium mapping for grain $\mathrm{Fe}$ and $\mathrm{Zn}$ enhancing QTLs useful for nutrient dense rice breeding. BMC plant Biology 20(1):57.

Pradhan SK, Pandit E, Pawar S, Pradhan A, Behera L, Das SR, Pathak H (2020a) Genetic regulation of homeostasis, uptake, biofortification and efficiency enhancement of iron in rice. Environmental and Experimental Botany 177:104066. DOI: 10.1016/j.envexpbot.2020.104066.

Prigge V, Melchinger AE, Dhillon BS, Frisch M (2009) Efficiency gain of marker-assisted backcrossing by sequentially increasing marker densities over generations. Theoretical and Applied Genetics 119(1): 23-32.

Rao DS, Neeraja CN, Babu PM, Nirmala B, Suman K, Rao LVS, Surekha K, Raghu P, Longvah T, Surendra P,00 Kumar R, Babu VR, Voleti SR (2020) Zinc biofortified rice varieties: challenges, possibilities, and progress in India. Frontiers in Nutrition 7:26. doi.org/10.3389/fnut.2020.00026.

Schlotterer C (2000) Evolutionary dynamics of microsatellite DNA. Chromosoma 109:365-371.

Servin B, Hospital F (2002) Optimal positioning of markers to control genetic background in marker-assisted backcrossing. Journal of Heredity 93: 214-217.

Shivani D, Suman K, Madhubabu P, Rathod R, Cheralu C, Shankar VG, Neeraja CN (2020) Parental polymorphism in ironand zinc-rich rice varieties (Swarna and type 3) using ssr markers. Applied Biological Research 22(1): 69-75.

Swamy BM, Kaladhar K, Shobha Rani N, Prasad GSV, Viraktamath BC, Reddy GA, Sarla N (2012) QTL analysis for grain quality traits in $2 \mathrm{BC}_{2} \mathrm{~F}_{2}$ populations derived from crosses between Oryza sativa cv Swarna and 2 accessions of $\mathrm{O}$. nivara. Journal of Heredity 103(3): 442-452.

Temnykh S, DeClerk G, Lukashova A, Lipovich L, Cartinhour S, McCouch S (2001) Computational and experimental analysis of microsatellites in rice (Oryza sativa L.): frequency, length variation, transposon associations, and genetic marker potential. Genome Research 11:1441-1452.

Temnykh S, Park WD, Ayres N, Cartinhour S, Hauck N, Lipovich L, Cho YG, Ishii T, McCouch SR (2000) Mapping and genome organization of microsatellite sequences in rice (Oryza sativa L.). Theoretical and Applied Genetics 100: 697-712.

The Rice Annotation Project Database (2019) The Rice Annotation Project Database available on https://rapdb.dna.affrc.go.jp/tools/blast accessed on 22 July, 2019.

Van Berloo R (2007) GGT: user manual Version 2.0. Wageningen (The Netherlands): Wegeningen University. Available on http:// www.plantbreeding.wur.nl/Software/ggt/ggt2_manual. Accessed on 23 July, 2019.

Van Berloo R (2008) GGT 2.0: Versatile Software for Visualization and Analysis of Genetic. Journal of Heredity 99(2): 232-236.

Virk P, Barry G (2009) Biofortified rice-towards combating human micronutrient deficiencies. International Rice Research Institute, DAPO Box 7777, Metro Manila, Philippines.

Vishalakshi B, Umakanth B, Usha G, Senguttuvel P, Prasad MS, Hari Y, Sudhakar P, Satya AK, Madhav MS (2020) Validation of Markers Linked to Yield under Drought, Blast Resistance and Assessment of Polymorphism among the Donors for Use in Marker Assisted Selection in Rice. Current Journal of Applied Science and Technology 181-190.

Waghmare SG, Sindhumole P, Shylaja MR, Mathew D, Francies RM, Abida PS, Sajini S (2018) Analysis of simple sequence repeat (SSR) polymorphism between N22 and Uma rice varieties for marker assisted selection. Electronic Journal of Plant Breeding 9(2): 511-517.

Yerva SR, Singh SK, Singh DK, Habde S, Behera PP (2018) Parental polymorphic survey for high $\mathrm{Zn}$ and $\mathrm{Fe}$ content in grains of rice (Oryza sativa. L) using SSR markers. Journal of Pharmacognosy and Phytochemistry 7(4): 1362-1367.

Zhang YY (2005) The analysis of genetic diversity for indica local rice from different place of China. MSc thesis. The Chinese Academy of Agricultural Sciences 